Elsevier required licence: (C) <2018>. This manuscript version is made available under the CC-BY-NC-ND 4.0 license http://creativecommons.org/licenses/by-nc-nd/4.0/ 


\title{
A Hybrid Evolutionary Preprocessing Method for Imbalanced Datasets
}

\author{
Ginny Y. Wong*, Frank H.F. Leung*, Sai-Ho Ling**
}

\begin{abstract}
Imbalanced datasets are commonly encountered in real-world classification problems. As many machine learning algorithms are originally designed for wellbalanced datasets, re-sampling has become an important step to pre-process imbalanced data. It aims at balancing the datasets by increasing the samples of the smaller class or decreasing the samples of the larger class, which are known as over-sampling and under-sampling respectively. In this paper, a sampling strategy based on both over-sampling and under-sampling is proposed, in which the new samples of the smaller class are created based on fuzzy logic. Improvement of the datasets are done by the evolutionary computational method of Cross-generational elitist selection, Heterogeneous recombination and Cataclysmic mutation (CHC)

\footnotetext{
${ }^{*}$ Centre for Signal Processing, Dept. of Electronic and Information Engg., Hong Kong Polytechnic University, Hung Hom, Hong Kong.

** Centre for Health Technologies, Faculty of Engineering and Information Technology, University of Technology Sydney, NSW, Australia.

Email addresses: ginnyyk .wong@connect . polyu.hk (Ginny Y. Wong), frank-h-f . leung@polyu . edu . hk (Frank H.F. Leung), steve. ling@uts . edu . au (Sai-Ho Ling)
} 
that under-samples both the minority and majority samples. As a result, a hybrid preprocessing method is proposed to re-sample imbalanced datasets. The evaluation is done by applying the Support Vector Machine (SVM), C4.5 decision tree and nearest neighbor rule to train a classification model from the re-sampled training sets. From the experimental results, it can be seen that our proposed method improves both the F-measure and AUC. The over-sampling rate and complexity of the classification model are also compared. Our proposed method is found to be superior to all other methods under comparison, and is more robust in different classifiers.

\section{Introduction}

The classification of imbalanced datasets is a popular topic in recent years [22] and [27]. Most of the machine learning tools, such as neural networks and support vector machines, are originally designed for well-balanced datasets. If the dataset is imbalanced, the performance of the classifier can be poor. The reason for this is apparent. For example, considering a dataset with $99 \%$ of data from class A and only $1 \%$ of data from class B, the accuracy is $99 \%$ if the classifier ignores the data from class B and labels the whole dataset as class A. It is already very hard to achieve an accuracy above $99 \%$ by using most of the learning algorithms. However, the minority class of datasets is usually more important and meaningful. For example, there are much less samples of people with a particular disease than 
those of healthy people in a medical problem. If a classifier is needed to label whether some people are infected or not, it is obvious that the minority class (people with a particular disease) is the class of interest.

Problems with imbalanced datasets can be easily found in the real world, such as intrusion detection [9], speech recognition [26], identification of power distribution fault causes [41], and bioinformatics problems [16]. There are two main approaches to solve the problems caused by imbalanced datasets. One is the data level approach and the other is the algorithm level approach. The data level approaches [3], [8], [18], and [28] include balancing the class distribution by oversampling the minority class or under-sampling the majority class. The algorithm level approaches improve the existing machine learning methods by adjusting the probabilistic estimate [38], modifying the cost per class [32], adding some penalty constants [25], or learning from one class instead of two classes [35] and [30].

Many experiments [12], [15], and [42] show that re-sampling is a good data level approach to handle imbalanced data. Moreover, it is more flexible as it does not depend on the chosen classifier. Therefore, we focus on re-sampling in this paper. There are three main types of strategies for re-sampling data. The first one is over-sampling, which can be done randomly or by the method of Synthetic Minority Over-sampling Technique (SMOTE) [8]. The second one is under-sampling, which includes Tomek links [37] and Neighborhood Cleaning Rule (NCL) [24]. The last one is the hybrid method, which combines the two previous methods 
(over-sampling and under-sampling methods).

The importance of designing sampling strategies has been discussed in [31], which may affect the successful learning of different classes. Hybrid re-sampling methods, reportedly, have advantage on treating datasets with a high imbalanced ratio [3] and [6]. Although some hybrid methods [3], [34], and [40] have been proposed to reduce the over-generalization problem from over-sampling methods, most of these methods are based on SMOTE and the results may be limited by the synthetic samples of SMOTE. Therefore, a hybrid re-sampling method is proposed in this paper. Fuzzy logic, which is a useful tool to treat imbalanced datasets [12], is used to over-sample the minority class samples instead of SMOTE. A fuzzy rule base is formed based on the samples of the minority class. Then, a rule is selected randomly with reference to the effectiveness of each rule. The selected rule is used as the criteria to generate a new sample of the minority class. The above steps will repeat until the sizes of the majority class and minority class are the same.

However, the large over-sampled training dataset will increase the complexity of the classification model and decrease the efficiency of the learning algorithm. It will also cause over-generalization easily, especially for some noisy dataset. This is because the decision boundary could become narrow or the overlapping area between the majority class and minority class could become large after the over-sampling. Therefore, an evolutionary algorithm (EA) is applied to both the 
synthetic samples and majority samples to under-sample the dataset. The chosen EA is the $\mathrm{CHC}$ (Cross-generational elitist selection, Heterogeneous recombination and Cataclysmic mutation) algorithm [11] since it shows the ability of selecting the most representative instances among many algorithms studied in [5].

Experiments are carried out to compare our proposed method with three SMOTEextended over-sampling methods, four hybrid re-sampling methods and one undersampling method. They are SMOTE, Safe-Level-SMOTE [4], Adaptive Synthetic Sampling [21], SMOTE+Tomek Links [3], SMOTE+Rough Set [34], SMOTE+CHC (sCHC) [40], agglomerative hierarchical clustering [10], and EUSCHC [14]. 44 imbalanced datasets from UCI Repository [2] are used in the experiments. The Support Vector Machine (SVM) [7], C4.5 decision tree [33], and nearest neighbor rule $(1 \mathrm{NN})$ are used as the tools for reaching a classification model for each resampled dataset so as to evaluate each re-sampling method. The evaluation measures are based on F-measure and area under the receiver operating characteristic curve (AUC). Although there exists many hybrid pre-processing methods, only some of them are like our method that consider and focus on the data size. In this paper, $\mathrm{CHC}$ is used to reduce the data size and achieve a good performance. Additionally, the proposed method enhances the performance in the over-sampling stage by taking advantage of the fuzzy rule base.

This paper is organized as follows: In Section 2, some preprocessing methods and $\mathrm{CHC}$ are reviewed. Section 3 presents the details of the proposed re-sampling 
strategy and the evaluation method. To show the effectiveness of our proposed approach, the comparisons with other methods and the results are discussed in Section 4. A conclusion is drawn in Section 5.

\section{Previous Work}

This section describes some previous works about re-sampling methods, which will be used to compare with our proposed method in the experiments later. The ideas about $\mathrm{CHC}$ will also be discussed.

\subsection{Re-sampling Methods}

As discussed in the previous section, there are three main strategies for resampling data.

\subsubsection{Over-sampling Methods}

Some instances are produced for the minority class to balance the class distribution. The simplest one is a non-heuristic method (random over-sampling) that replicates samples of the original minority class to generate the new instances. This method causes over-fitting easily since the new instances copy exactly from the original minority class. Synthetic Minority Over-sampling Technique (SMOTE) [8] is a well-known method which creates the new instances by interpolating several minority samples that join together. This method makes use 
of each minority class sample and inserts synthetic samples along the line segments joining any/all of the $k$ minority class nearest neighbors to over-sample the minority class. An example is shown in Fig. 1. Five nearest neighbors are used in it, where $x_{i}$ is a selected sample of minority class, $x_{i 1}$ to $x_{i 5}$ are the 5 nearest neighbors of $x_{i}$ and $s_{1}$ to $s_{5}$ are the synthetic samples created by interpolation. If the degree of over-sampling required is $300 \%$, three synthetic examples are selected randomly from $s_{1}$ to $s_{5}$.

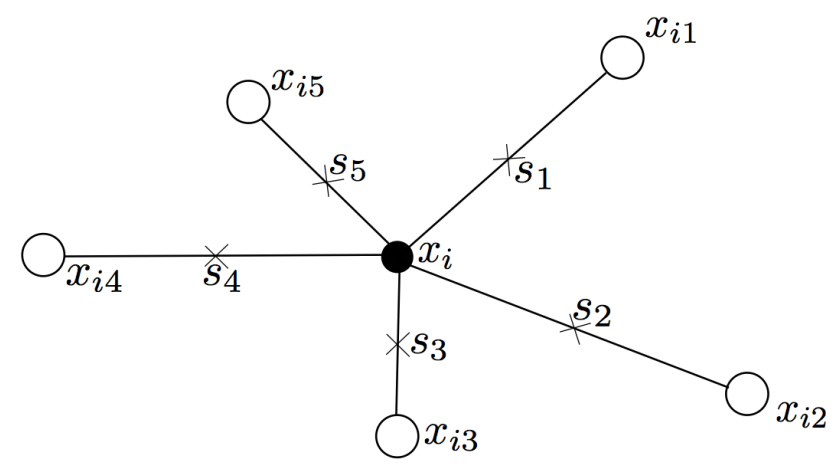

Figure 1: Example of SMOTE with 5 nearest neighbors.

Since the synthetic samples provide a less specific and larger decision region, the over-fitting problem can be reduced. However, this method may introduce more minority synthetic samples in the area of majority class where the minority class is very sparse with respect to the majority class. This causes the problem of over-generalization, which means the decision boundary is very narrow or there is a large overlapping area between the majority class and minority class. Therefore, some methods are developed based on SMOTE to overcome this limitation, such 
as Borderline-SMOTE (sBorder) [19], Adaptive Synthetic Sampling (ADASYN) [21], Safe-Level-SMOTE (sSafe) [4], and SPIDERS [29].

\subsubsection{Under-sampling Methods}

Some instances of majority class are eliminated in order to balance the class distribution. The simplest method is random under-sampling (RUS), which aims to balance the datasets by randomly removing samples of the majority class. However, this method may easily remove some useful data. The other representative methods include (i) condensed nearest neighbor rule (CNN) [20], which eliminates the majority class samples that are distant from the decision border, (ii) Tomek links (TL) [37], which edits out noisy and borderline majority class samples, (iii) one-sided selection (OSS) [23], which is an integrated method of TL and $\mathrm{CNN}$, and (iv) neighborhood cleaning rule (NCL) [24], which is based on the Wilson's Edited Nearest Neighbor Rule (ENN) [39] to remove the majority class samples that lead to misclassification.

\subsubsection{Hybrid Methods}

Although both over-sampling and under-sampling can balance the class distribution, different drawbacks like over-generalization and removal of useful data are also introduced. Therefore, some hybrid methods are developed to combine SMOTE and under-sampling as a data cleaning method to reduce the problem. Example hybrid methods include SMOTE+Tomek links (sTL), which uses TL 
to remove samples of both classes to increase the area of decision border, and SMOTE+ENN (sENN) [3], which uses ENN to remove the samples that are misclassified by their nearest neighbors. Rough set theory (sRST) [34] and evolutionary algorithm (sCHC) [40] have also been applied on SMOTE to select the samples to increase the accuracy of classification.

Most of the above hybrid methods make use of SMOTE to perform oversampling. Clustering techniques are also developed to perform under-sampling and over-sampling, such as agglomerative hierarchical clustering (AHC) [10].

\section{2. $\mathrm{CHC}$ [11]}

$\mathrm{CHC}$ is a kind of EAs that combines a selection strategy with a highly disruptive recombination operator. To avoid premature convergence and maintain diversity, incest prevention and cataclysmic mutation are introduced. The process of $\mathrm{CHC}$ can be described as follows. Firstly, a population set of chromosomes $P$ is created. Each chromosome $p_{i}=\left(p_{i 1}, p_{i 2}, \ldots, p_{i n}\right)$ is an $n$-dimensional vector, which is a set of genes, where $p_{i j}$ is the $j$ th gene value $(j=1,2, \ldots, n)$ of the $i$ th chromosome in the population $(i=1,2, \ldots, m) ; m$ is the population size and $n$ is the number of genes.

Secondly, the chromosomes are evaluated by a defined fitness function. The form of fitness function depends on the application. Thirdly, an intermediate population set of chromosomes $C$, which is of the same size as $P$, is generated by 
copying all members of $P$ in a random order.

Then, a uniform crossover (HUX) operator is applied on $C$ to form $C^{\prime}$. HUX exchanges half of the genes randomly between the chromosomes one by one to form $C^{\prime}$. CHC also uses an additional method for incest prevention. Before applying HUX to the chromosomes, the Hamming distance between them is calculated. If half of that distance is larger than a difference threshold $d$, HUX is applied; otherwise these two chromosomes are deleted from $C$. Therefore, the size of $C^{\prime}$ may be smaller than that of $P$ or $C$. The initial threshold $d$ is set at $n / 4$. After $C^{\prime}$ has formed, it is evaluated by the fitness function and an elitist selection is taken. Only the best chromosomes from both $P$ and $C^{\prime}$ are selected to form the offspring population in the next generation. If the offspring population is the same as $P$, the difference threshold $d$ is decreased by one.

$\mathrm{CHC}$ is different from the traditional genetic algorithm. Mutation is not performed at the recombination stage. $\mathrm{CHC}$ performs partial reinitialization (divergence) when the search becomes trapped (i.e., the difference threshold $d$ becomes zero and no new offspring population is formed for several generations). The population is reinitialized, based on the best chromosome, by changing the elements' values randomly with a user-defined divergence rate $D_{\text {rate }}$. For example, if $D_{\text {rate }}$ equals to 0.35 , the values of $35 \%$ elements will be changed randomly. The search is then resumed with a new difference threshold $d=D_{\text {rate }} *\left(1-D_{\text {rate }}\right) * n$. This process is called cataclysmic mutation. 
$\mathrm{CHC}$ has shown the ability of selecting the most representative instances among the other algorithms studied in [5]. Therefore, it is chosen as the algorithm to improve the outcome of over-sampling in this paper.

\section{Methodology}

In this section, the proposed hybrid preprocessing method and the evaluation methods used in this paper are discussed. The proposed method involves two stages. The minority samples of the training sets are firstly over-sampled based on fuzzy logic to form a fuzzy rule base (FRB). To improve the performance, $\mathrm{CHC}$ is then implemented to reduce both the synthetic samples and majority samples.

\subsection{Fuzzy Rule Base (FRB)}

In this paper, let the positive class be the minority class and only $\lambda$ training samples $\left(X_{\alpha}\right)$ of positive class are considered, where $X_{\alpha}=\left(x_{\alpha 1}, \ldots, x_{\alpha \gamma}\right)$ is an $\gamma$-dimensional vector, $\alpha=1,2, \ldots, \lambda$ and $x_{\alpha \beta}$ is the $\beta$ th attribute value $(\beta=$ $1,2, \ldots, \gamma)$ of the $\alpha$ th training sample. The $\theta$ th fuzzy if-then rule is written as follows:

$$
\begin{array}{r}
\text { Rule } \theta: \text { IF } z_{1} \text { is } A_{1}^{\theta} \text { AND } \ldots \text { AND } z_{\gamma} \text { is } A_{\gamma}^{\theta} \\
\text { THEN class }=\text { positive with } w_{\theta}
\end{array}
$$

where $A_{\beta}^{\theta}$ is a fuzzy term of the $\theta$ th rule corresponding to the attribute $z_{\beta}, \beta=$ $(1,2, \ldots, \gamma)$ and $z=\left(z_{1}, z_{2}, \ldots, z_{\gamma}\right)$ is a $\gamma$-dimensional attribute vector, and $w_{\theta}$ 
is the rule weight. The regular triangular membership functions are used for the fuzzy terms. In this paper, the fuzzy terms $A_{\beta}^{\theta}$ are derived based on the samples of positive class. The minimum and maximum values of each attribute are first found. The fuzzy terms are the triangular membership functions within the range of each attribute. The fuzzy terms also depend on the number of labels. Since regular triangular membership functions are used, the fuzzy terms are distributed evenly within the range of each attribute.

The fuzzy rules are generated based on the samples of positive class. For each sample, the label with the highest membership value is selected to form the corresponding rule for each attribute. The maximum number of rules depends on the number of labels and attributes.

The rule weight $w_{\theta}$ is used to reflect the degree of matching of each fuzzy rule over all the positive samples, so that the importance of each rule can be evaluated. First, the fuzzy value of each sample is calculated. The fuzzy value of $X_{\alpha}$ for the $\theta$ th fuzzy rule is defined as follows:

$$
\mu_{A^{\theta}}\left(X_{\alpha}\right)=T\left(\mu_{A_{1}^{\theta}}\left(x_{\alpha 1}\right), \ldots, \mu_{A_{\gamma}^{\theta}}\left(x_{\alpha \gamma}\right)\right),
$$

where the product T-norm is used. The rule weight $\left(w_{\theta}\right)$ is calculated by adding all the fuzzy values of samples.

$$
w_{\theta}=\sum_{\alpha=1}^{\lambda}\left(\mu_{A^{\theta}}\left(X_{\alpha}\right)\right) .
$$


After the rule base of the positive class is generated, the rules are randomly drawn based on the rule weight. The rule with a higher rule weight will have a higher probability to be chosen. Then, a new sample is generated within the area of the selected rule. These processes are repeated until the number of positive samples is the same as that of the negative samples.

To illustrate the idea more clearly, Fig. 2 shows the distribution of two classes with two attributes as an example of the formulation of fuzzy rules. The x-axis and y-axis govern the values of the two different attributes and regular triangular membership functions with five labels are used. The circle dots correspond to the negative class and the square dots correspond to the positive class. The dashed lines show the minimum or maximum value of the corresponding attribute of the positive samples. As only the attribute vectors of the positive class are considered to generate fuzzy rules, totally ten rules can be formed in this example: 
Rule 1: IF $z_{1}$ is $A_{1}^{1}=L 1 \_1$ AND $z_{2}$ is $A_{2}^{1}=L 2 \_4 . \quad$ THEN class = positive with 0.897

Rule 2: IF $z_{1}$ is $A_{1}^{2}=L 1 \_2$ AND $z_{2}$ is $A_{2}^{2}=L 2 \_3 . \quad$ THEN class = positive with 1.147

Rule 3: IF $z_{1}$ is $A_{1}^{3}=L 1 \_2$ AND $z_{2}$ is $A_{2}^{3}=L 2 \_4 . \quad$ THEN class = positive with 1.508

Rule 4: IF $z_{1}$ is $A_{1}^{4}=L 1 \_3$ AND $z_{2}$ is $A_{2}^{4}=L 2 \_3 . \quad$ THEN class = positive with 1.230

Rule 5: IF $z_{1}$ is $A_{1}^{5}=L 1 \_3$ AND $z_{2}$ is $A_{2}^{5}=L 2 \_4 . \quad$ THEN class = positive with 2.344

Rule 6: IF $z_{1}$ is $A_{1}^{6}=L 1 \_3 \mathrm{AND} z_{2}$ is $A_{2}^{6}=L 2 \_5 . \quad$ THEN class = positive with 1.607

Rule 7: $\mathrm{IF} z_{1}$ is $A_{1}^{7}=L 1_{\_} 4 \mathrm{AND} z_{2}$ is $A_{2}^{7}=L 2 \_1 . \quad$ THEN class = positive with 0.727

Rule 8: IF $z_{1}$ is $A_{1}^{8}=L 1 \_4$ AND $z_{2}$ is $A_{2}^{8}=L 2 \_4 . \quad$ THEN class = positive with 1.319

Rule 9: IF $z_{1}$ is $A_{1}^{9}=L 1 \_4$ AND $z_{2}$ is $A_{2}^{9}=L 2 \_5 . \quad$ THEN class = positive with 1.731

Rule 10: $\mathrm{IF} z_{1}$ is $A_{1}^{10}=L 1 \_5$ AND $z_{2}$ is $A_{2}^{10}=L 2 \_4 . \quad$ THEN class = positive with 1.399

where $z_{1}$ and $z_{2}$ represent Attribute 1 and Attribute 2 for the $\mathrm{x}$-axis and $\mathrm{y}$-axis respectively in Fig. 2, $L 1 \_i$ is the $i$-th label of $z_{1}$ attribute, $L 2 \_i$ is the $i$-th label of $z_{2}$ attribute. Rule 5 has the highest rule weight and rule 7 has the lowest rule weight in this example.

For generating the synthetic samples, a rule out of these ten rules is chosen with the probability of selection depending on the rule weight. Then, this rule sets the criteria of the highest and lowest value of each attribute. The new sample is generated randomly within these criteria. This process is repeated until the num- 
ber of the positive class is the same as that of the negative class. Fig. 3 shows the samples distribution after over-sampling. The triangle dots represent the synthetic samples. It is found that the spread of the synthetic samples is similar to that of the original positive samples (shown as the square dots). The synthetic samples in Fig. 3 are dense in the area of rule 5.

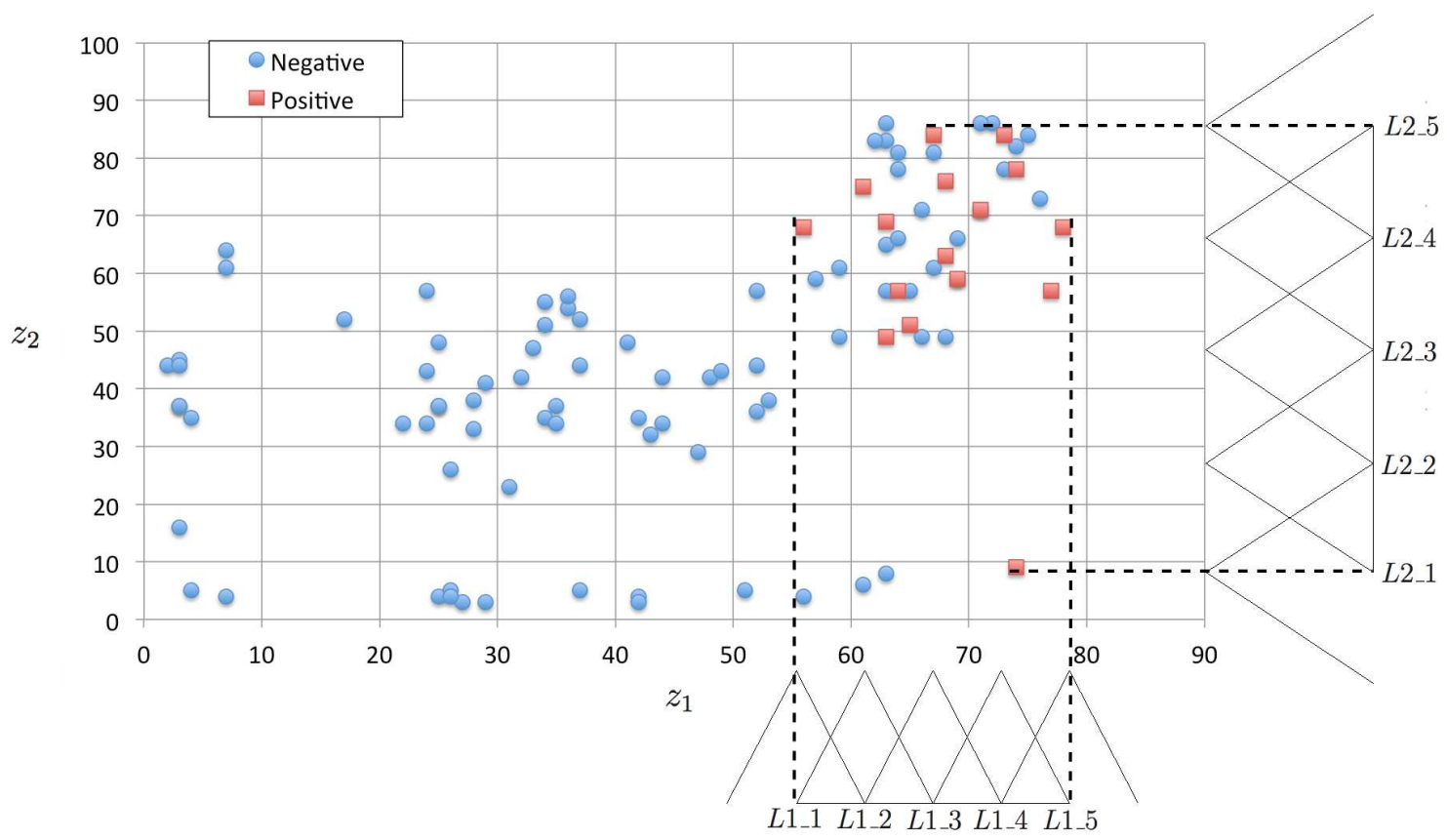

Figure 2: Example of the distribution of imbalanced dataset. The y-axis represents the values of $z_{2}$ and $\mathrm{x}$-axis represents the value of $z_{1}$.

\subsection{Setting of $\mathrm{CHC}$}

After the over-sampling, the number of minority samples is the same as that of majority samples and $\mathrm{CHC}$ is then applied. There are two important issues that need to be addressed before the algorithm is employed: the representation of each 


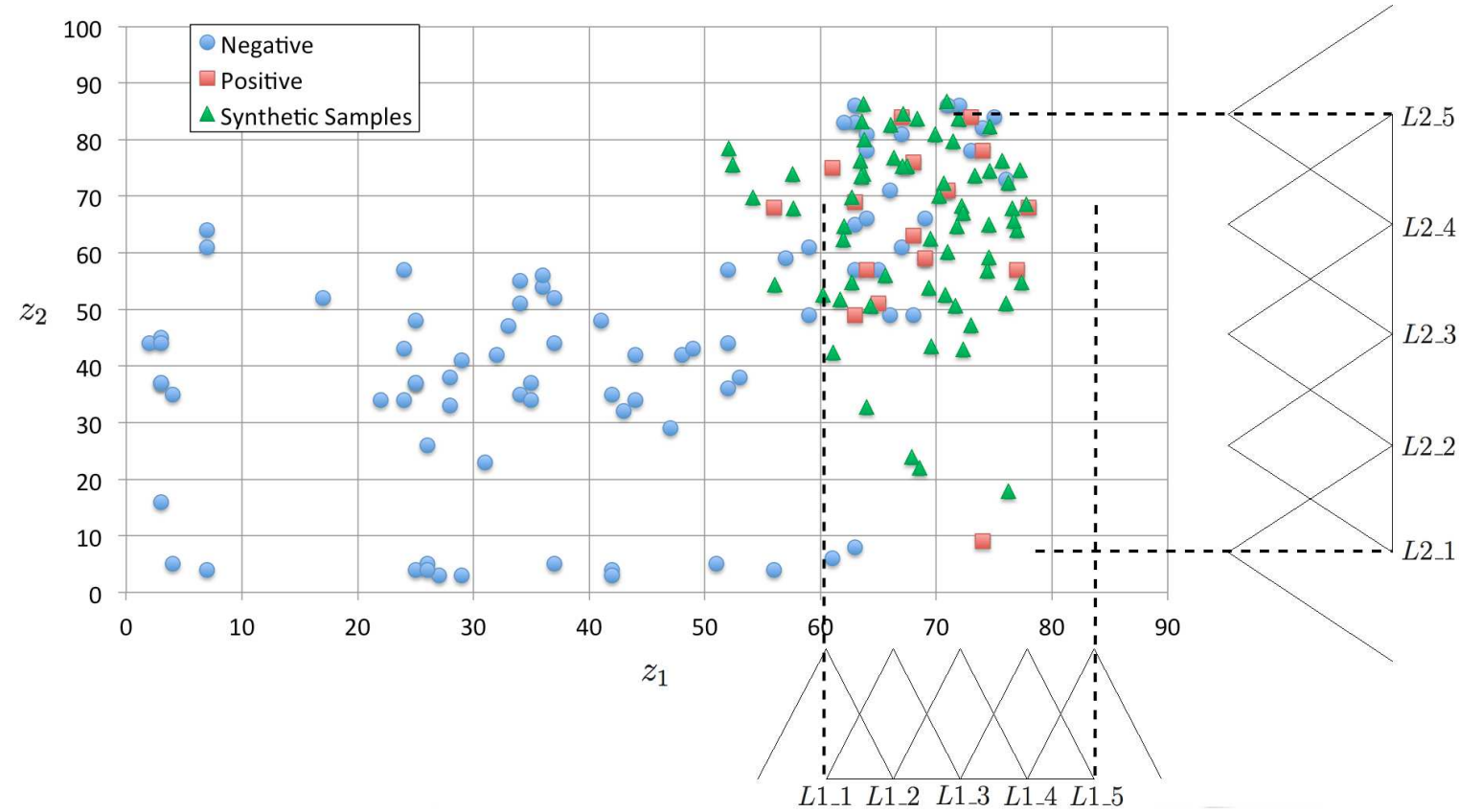

Figure 3: Distribution of the samples after over-sampling. The y-axis represents the values of $z_{2}$ and $\mathrm{x}$-axis represents the value of $z_{1}$.

chromosome and the definition of fitness function. Fig. 4 shows the block diagram of the process of $\mathrm{FRB}+\mathrm{CHC}$.

\subsubsection{Chromosome Representation}

$\mathrm{CHC}$ is used to reduce the synthetic samples as well as the majority class samples. Therefore, the chromosomes are to represent subsets of these samples. It can be carried out by a binary representation. Each chromosome is an $n$-dimensional vector. In this section, $n$ is the number of synthetic samples plus majority class samples. Each vector element shows whether the corresponding sample exists in the subset of the training set or not. Therefore, there are two possible values for 


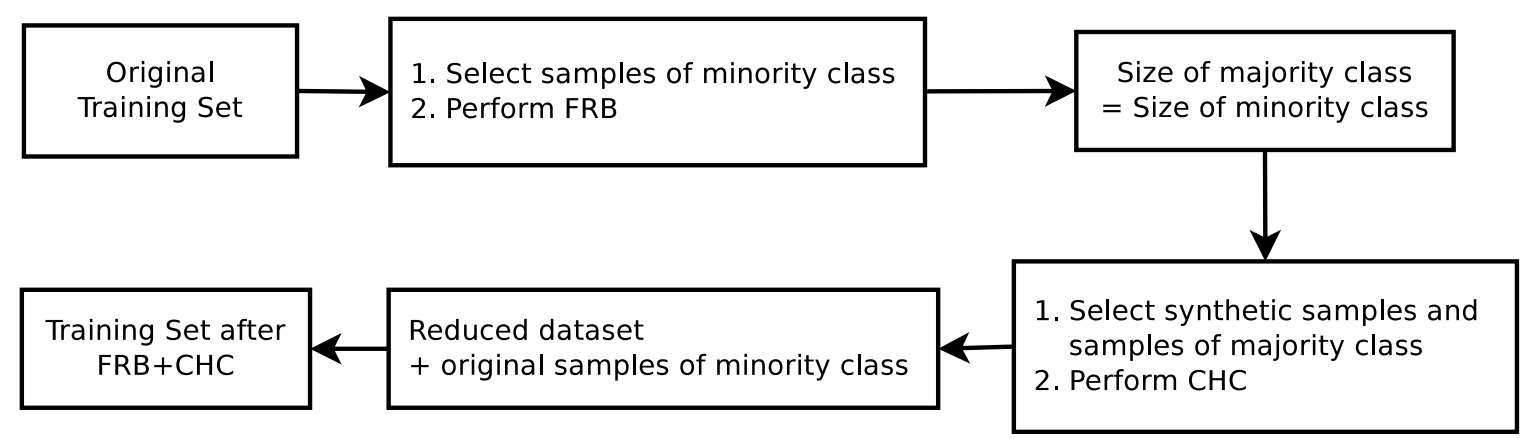

Figure 4: Block Diagram of FRB+CHC.

each element: 0 and 1 . If the value is 1 , the corresponding sample is included in the subset of the training set. If the value is 0 , the sample does not exist in the subset.

\subsubsection{Fitness function}

In this study, the k-NN classifier is used as the evaluation method of $\mathrm{CHC}$ to obtain the subset with the highest classification rate. Normally, accuracy (ratio of correctly classified samples to total number of samples) would be used as the measure of classification rate. However, it may cause difficulty for the imbalanced datasets when doing testing later since the correct classification rate of the majority samples may affect the accuracy more significantly than that of the minority samples. Therefore, some other measures are used in this paper. These measures are commonly employed to analyze problems with imbalanced datasets.

Firstly, precision and recall are introduced [17]. Their definitions are given as 
follows:

$$
\begin{aligned}
\text { Precision } & =\frac{T P}{T P+F P} \\
\text { Recall } & =\frac{T P}{T P+F N}
\end{aligned}
$$

where $T P$ is the number of true positives, $F P$ is the number of false positives and $F N$ is the number of false negatives. A high value of precision indicates that the predicted positive samples are most likely relevant. A high value of recall indicates that most of the positive samples can be predicted correctly.

A popular evaluation metric for imbalanced problems is $F$ - measure [17], which is a function of precision and recall. In principle, $F-$ measure represents a harmonic mean between precision and recall. A high value of $F-$ measure means both the precision and recall values are high and do not differ very much. It is an important measure for imbalanced datasets since a high value of it can imply that the method classifies the positive samples correctly at a high rate with little misclassified negative samples. It is defined as follows:

$$
F-\text { measure }=\frac{2 * \text { Precision } * \text { Recall }}{\text { Precision }+ \text { Recall }}
$$

The area under the receiver operating characteristic curve (AUC) is also commonly used to measure the performance of classification. The AUC measure [13] is the probability of correctly identifying a random sample, and it can be defined 
as follows:

$$
A U C=\frac{1+\text { Recall }-F P_{\text {rate }}}{2}
$$

where Recall is defined in (5) and $F P_{\text {rate }}=\frac{F P}{F P+T N}, T N$ is the number of true negatives. $F P_{\text {rate }}$ defines the percentage of true negatives cases misclassified as positives. A high value of $A U C$ implies small values of $F N$ and $F P$, meaning that the corresponding classifier is effective.

Since both $F-$ measure and $A U C$ are important measures on imbalanced datasets, a multi-objective fitness function is used here. The chromosome with both higher values of $F-$ measure and $A U C$ obviously has a higher rank. If a chromosome $X$ has a higher value of $F-$ measure $\left(F_{X}>F_{Y}\right)$ and a lower value of $A U C\left(A_{X}<A_{Y}\right)$ than that of chromosome $Y$, the difference between the chromosomes' $F-$ measure $\left(\left|F_{X}-F_{Y}\right|\right)$ and the difference between the chromosomes' $A U C\left(\left|A_{X}-A_{Y}\right|\right)$ will be compared. If $\left|F_{X}-F_{Y}\right|>\left|A_{X}-A_{Y}\right|$, chromosome $X$ will be regarded as a better one; otherwise chromosome $Y$ will be regarded as a better one. The above setting is also applied in $\mathrm{sCHC}$ for the comparison in Section 4.

\subsection{Evaluation}

\subsubsection{F-measure and AUC measures}

To show the performance of our proposed method, $F$ - measure in (6) and $A U C$ in (7) are used. The main drawback of over-sampling or hybrid sampling 
methods is that the number of training samples are increased greatly. This may cause the increase of complexity of the learning model. Therefore, the oversampling rates of different methods are also compared. Define:

$$
\text { Rate }_{\text {over }}=\frac{\left(N_{\text {sampled }}-N_{\text {original }}\right)}{N_{\text {original }}} * 100 \%
$$

where $N_{\text {sampled }}$ is the number of samples in the re-sampled training set and $N_{\text {original }}$ is the number of samples in the original training set. The over-sampling rate in (8) shows the increase rate of the number of the training samples. When a support vector machine is used to form the classification mode, the increase rate of the support vectors can be used to evaluate the complexity of the learning model. This rate is calculated based on the support vectors generated.

$$
\text { Rate }_{S V}=\frac{\left(S V_{\text {sampled }}-S V_{\text {original }}\right)}{S V_{\text {original }}}
$$

where $S V_{\text {sampled }}$ is the number of support vectors trained by the re-sampled training set and $S V_{\text {original }}$ is the number of support vectors trained by the original training set. It should be noted that the $\mathrm{CHC}$ fitness evaluation for data size reduction (by k-NN) and the training of the classification model based on the resampled data (by SVM) are two separated processes. K-NN is used in the fitness evaluation because it is simple with minimal computation effort. SVM is a commonly used method to obtain the classification model. 


\section{Experimental Study}

In this section, we present the experiments that are carried out to compare our proposed method with other hybrid sampling methods and the $\mathrm{CHC}$ undersampling method. The datasets used can be found in UCI Repository [2].

The experiments involve different kinds of hybrid methods, including SMOTE, ADASYN, sTL, sSafe, sRST, sCHC, AHC and our proposed method, which is named as Fuzzy Rule Base+CHC (FRB+CHC). $\mathrm{CHC}$, which is used as an undersampling method in [14] (EUSCHC), is also compared in the experiment. To measure the performance of the preprocessing methods, the same learning tool should be applied among all the experiments. In this study, three different tools are used. They are Support Vector Machine (SVM), 1 Nearest Neighbor (1NN), and $\mathrm{C} 4.5$ decision tree. The programs of all testing methods and the learning tools are based on KEEL, which is an open source software available in the Web [1]. $F-$ measure and $A U C$ are used as measures to analyze the results. The average values of these measures for each method will be calculated. As the expansion of re-sampled training datasets may increase the computational time and complexity of the classification model, the over-sampling rate and the number of support vectors formed from SVM will also be compared. 


\subsection{Datasets}

To study the methods on different datasets, 44 datasets with different imbalance ratio (IR) are chosen. IR is the ratio of the number of majority class to the number of minority class. Table 1 shows the details of the selected datasets, where the number of samples $\left(N_{\text {samp }}\right)$, the number of attributes $\left(N_{\text {attr. }}\right)$, the distribution of the minority and majority classes, and IR for each dataset can be found.

Table 1: Details of the Selected Imbalanced Datasets.

\begin{tabular}{|c|c|c|c|c|}
\hline Dataset & $\boldsymbol{N}_{\text {samp. }}$ & $\boldsymbol{N}_{\text {attr. }}$ & Min., Maj.(\%) & IR \\
\hline ecoli034vs5 & 200 & 7 & $(10,90)$ & 9 \\
yeast2vs4 & 514 & 8 & $(9.92,90.08)$ & 9.08 \\
ecoli067vs35 & 222 & 7 & $(9.91,90.09)$ & 9.09 \\
ecoli0234vs5 & 202 & 7 & $(9.9,90.1)$ & 9.1 \\
glass015vs2 & 172 & 9 & $(9.88,90.12)$ & 9.12 \\
yeast0359vs78 & 506 & 8 & $(9.88,90.12)$ & 9.12 \\
yeast0256vs3789 & 1004 & 8 & $(9.86,90.14)$ & 9.14 \\
yeast02579vs368 & 1004 & 8 & $(9.86,90.14)$ & 9.14 \\
ecoli046vs5 & 203 & 6 & $(9.85,90.15)$ & 9.15 \\
ecoli01vs235 & 244 & 7 & $(9.83,90.17)$ & 9.17 \\
ecoli0267vs35 & 224 & 7 & $(9.82,90.18)$ & 9.18 \\
glass04vs5 & 92 & 9 & $(9.78,90.22)$ & 9.22 \\
ecoli0346vs5 & 205 & 7 & $(9.76,90.24)$ & 9.25 \\
ecoli0347vs56 & 257 & 7 & $(9.73,90.27)$ & 9.28 \\
yeast05679vs4 & 528 & 8 & $(9.66,90.34)$ & 9.35 \\
vowel0 & 988 & 13 & $(9.01,90.99)$ & 9.98 \\
ecoli067vs5 & 220 & 6 & $(9.09,90.91)$ & 10 \\
\hline
\end{tabular}




\begin{tabular}{|c|c|c|c|c|}
\hline Dataset & $\boldsymbol{N}_{\text {samp. }}$ & $\boldsymbol{N}_{\text {attr. }}$ & Min., Maj.(\%) & IR \\
\hline glass016vs2 & 192 & 9 & $(8.85,91.15)$ & 10.29 \\
ecoli0147vs2356 & 336 & 7 & $(8.63,91.37)$ & 10.59 \\
led7digit02456789vs1 & 443 & 7 & $(8.35,91.65)$ & 10.97 \\
ecoli01vs5 & 240 & 6 & $(8.33,91.67)$ & 11 \\
glass06vs5 & 108 & 9 & $(8.33,91.67)$ & 11 \\
glass0146vs2 & 205 & 9 & $(8.29,91.71)$ & 11.06 \\
glass2 & 214 & 9 & $(7.94,92.06)$ & 11.59 \\
ecoli0147vs56 & 332 & 6 & $(7.53,92.47)$ & 12.28 \\
cleveland0vs4 & 177 & 13 & $(7.34,92.66)$ & 12.62 \\
ecoli0146vs5 & 280 & 6 & $(7.14,92.86)$ & 13 \\
shuttlec0vsc4 & 1829 & 9 & $(6.72,93.28)$ & 13.87 \\
yeast1vs7 & 459 & 7 & $(6.53,93.47)$ & 14.3 \\
glass4 & 214 & 9 & $(6.07,93.93)$ & 15.47 \\
ecoli4 & 336 & 7 & $(5.95,94.05)$ & 15.8 \\
page_blocks13vs4 & 472 & 10 & $(5.93,94.07)$ & 15.86 \\
abalone918 & 731 & 8 & $(5.65,94.25)$ & 16.4 \\
glass016vs5 & 184 & 9 & $(4.89,95.11)$ & 19.44 \\
shuttlec2vsc4 & 129 & 9 & $(4.65,95.35)$ & 20.5 \\
yeast1458vs7 & 693 & 8 & $(4.33,95.67)$ & 22.1 \\
glass5 & 214 & 9 & $(4.2,95.8)$ & 22.78 \\
yeast2vs8 & 482 & 8 & $(4.15,95.85)$ & 23.1 \\
yeast4 & 1484 & 8 & $(3.43,96.57)$ & 28.1 \\
yeast1289vs7 & 947 & 8 & $(3.16,96.84)$ & 30.57 \\
\hline
\end{tabular}




\begin{tabular}{|c|c|c|c|c|}
\hline Dataset & $\boldsymbol{N}_{\text {samp. }}$ & $\boldsymbol{N}_{\text {attr. }}$ & Min., Maj.(\%) & IR \\
\hline abalone19 & 4174 & 8 & $(0.77,99.23)$ & 129.44 \\
\hline
\end{tabular}

\subsection{Setup of Experiment}

For over-sampling, the rules of the minority samples are associated with regular triangular membership functions with five fuzzy terms. For CHC, the values of the parameters are:

- Population size: 50.

- Divergence rate: 0.35 .

- Threshold decreasing rate: 0.001 .

- $\mathrm{k}$ of k-NN classifier used as evaluation: 1.

- Number of evaluations: 5,000.

In this paper, SVM, $1 \mathrm{NN}$, and $\mathrm{C} 4.5$ are used to weigh the influence of each preprocessing method. For SVM, a radial basis function (RBF) is used as the kernel since a non-linear classification model is needed and RBF is a common kernel to handle this problem. The RBF is defined as follows:

$$
R B F=\exp \left(-\frac{1}{\sigma}\left\|\mathbf{x}_{i}-\mathbf{x}\right\|^{2}\right)
$$

where $\sigma>0$ is the parameter to determine the width of the radial basis function. It controls the flexibility of the classifier. When $\sigma$ decreases, the flexibility of 
the resulting classifier in fitting the training data increases, and this might lead to over-fitting easily. The value of $\sigma$ is set as 0.01 . The tradeoff between training error and margin of SVM is set as 100. The above values are chosen through experiments. For C4.5, the confidence level is set as 0.25 , the minimum number of item-sets per leaf is set to 2 and pruning is used as well to obtain the final tree. For $1 \mathrm{NN}$, the Euclidean distance metric is used.

A 5-fold cross validation model is used to compare the classification results from different preprocessing methods. Each dataset are first divided into five parts randomly. Four of them are combined to form a training set and the remaining subset forms a testing set. The process is then repeated five times, so that each subset is used once as a testing set. All the methods involve some random parameters, so five experiments are carried out for each 5-fold cross validation model and the average value are calculated as the results, i.e. totally 25 experiments were done.

\subsection{Results}

\subsubsection{F-measure and AUC measures}

Tables 2 and 3 show the SVM results on $F$ - measure and $A U C$ for each re-sampling method on the 44 datasets respectively. The results of the original datasets are shown in the second column and the best value for each dataset are highlighted in bold. The last row shows the average value of each sampling method for the datasets. The performance of the FRB over-sampling method 
are also included (in the rightmost column) for comparison with $\mathrm{FRB}+\mathrm{CHC}$. It can be seen that the average values of $F-$ measure and $A U C$ in both FRB and $\mathrm{FRB}+\mathrm{CHC}$ are higher than other methods. The performance of $\mathrm{sCHC}$ and $\mathrm{FRB}+\mathrm{CHC}$ are similar. This shows that $\mathrm{CHC}$ has good performance as a data cleaning method after over-sampling, especially for the results in $F$ - measure. The $A U C$ values of SMOTE, sTL, sSafe, sRST and sCHC are similar since they all use SMOTE to perform over-sampling. ADASYN gets the lowest average values of $F$-measure, which means the precision is low and the difference between precision and recall is large.

In this experiment, the performance of $\mathrm{FRB}$ and $\mathrm{FRB}+\mathrm{CHC}$ is very similar, which shows the advantages of FRB over the other hybrid or over-sampling methods. However, the data size will be very large if only FRB is used as the preprocessing method. $\mathrm{FRB}+\mathrm{CHC}$ can reduce the data size without a large effect to the performance. Therefore, only $\mathrm{FRB}+\mathrm{CHC}$ will be considered in the following section.

Table 4 shows the average rankings by means of $F-$ measure and $A U C$ using Friedman's method [36]. The highest value of each dataset is ranked as 1 . If a certain method obtains the ranking $3,6,2$, and 1 on four datasets, the average ranking is $(3+6+2+1) / 4=3$. Therefore, a lower average ranking indicates that the corresponding method is better among the other methods. FRB+CHC obtains the best ranking by $A U C$ and $\mathrm{sCHC}$ obtains the best ranking by $F-$ 
measure. Note that the highest average values of $A U C$ or $F-$ measure do not imply the best ranking results since the ranking shows the comparison results among all the methods of each dataset. For example, EUSCHC has the lowest $A U C$ average values but its ranking is better than ADASYN. Since EUSCHC is an under-sampling method, it easily ignores some useful samples of majority class. 
Table 2: SVM: Average F-measure of Testing Datasets among Different Sampling Methods.

\begin{tabular}{|c|c|c|c|c|c|c|c|c|c|c|c|}
\hline Dataset & Original & SMOTE & ADASYN & sTL & sSafe & SRST & sCHC & EUSCHC & AHC & FRB+CHC & FRB \\
\hline ecoli034vs5 & 0 & 0.5629 & 0.2667 & 0.5901 & 0.5578 & 0.5007 & 0.5054 & 0.6591 & 0.3111 & 0.5829 & 0.6337 \\
\hline yeast2vs4 & 0.6384 & 0.6824 & 0.5446 & 0.6683 & 0.6824 & 0.6787 & 0.6996 & 0.7418 & 0.6937 & 0.7015 & 0.6971 \\
\hline ecoli067vs35 & 0.0000 & 0.4540 & 0.3975 & 0.5122 & 0.4609 & 0.4447 & 0.5108 & 0.4758 & 0.3692 & 0.4308 & 0.6171 \\
\hline ecoli0234vs5 & 0.0000 & 0.5176 & 0.2917 & 0.5240 & 0.5012 & 0.4734 & 0.5577 & 0.6682 & 0.2667 & 0.6142 & 0.6462 \\
\hline glass0 & 0.0000 & 0.3094 & 0.3181 & 0.3103 & 0.3301 & 0.3419 & 0.2137 & 0.1015 & 0.2850 & 0.2049 & 0.2275 \\
\hline yeast 0359 vs78 & 0.3481 & 0.3541 & 0.2965 & 0.3379 & 0.3580 & 0.3529 & 0.4117 & 0.3481 & 0.3666 & 0.3470 & 0.3481 \\
\hline yeast $0256 v s 3789$ & 0.1782 & 0.5282 & 0.4391 & 0.5206 & 0.5286 & 0.5325 & 0.5624 & 0.6033 & 0.5263 & 0.5899 & 0.2589 \\
\hline yeast $02579 v s 368$ & 0.8152 & 0.7199 & 0.5213 & 0.7179 & 0.7189 & 0.7201 & 0.7437 & 0.7487 & 0.7264 & 0.7747 & 0.85 \\
\hline ecoli0 & 0.0000 & 0.3901 & 0.2000 & 0.3958 & 0.4084 & 0.4214 & 0.3827 & 0.6786 & 0.0667 & 0.5225 & 0.6584 \\
\hline ecoli01vs235 & 0.0000 & 0.4325 & 0.1648 & 0.4396 & 0.4352 & 0.4264 & 0.4844 & 0.5691 & 0.1385 & 0.4224 & 0.5536 \\
\hline ecoli0267vs35 & 0.0000 & 0.3158 & 0.2269 & 0.3257 & 0.2902 & 0.3253 & 0.3856 & 0.4035 & 0.1469 & 0.4592 & 0.5337 \\
\hline glass & 1.0000 & 0.8793 & 0.8679 & 0.8747 & 0.9228 & 0.9209 & 0.9933 & 0.7854 & 1.0000 & 0.9631 & 0.9655 \\
\hline ecoli03 & 0.0000 & 0.5446 & 0.3636 & 0.6397 & 0.5741 & 0.5642 & 0.5985 & 0.7382 & 0.3404 & 0.6766 & 0.6768 \\
\hline ecoli0347vs56 & 0.0000 & 0.5743 & 0.4743 & 0.5628 & 0.5576 & 0.5104 & 0.5913 & 0.6669 & 0.1846 & 0.5176 & 0.5913 \\
\hline yeast $05679 \mathrm{vs} 4$ & 0.0000 & 0.4327 & 0.4265 & 0.4282 & 0.4333 & 0.4250 & 0.5066 & 0.4996 & 0.4189 & 0.4786 & 0.5355 \\
\hline & 1.0000 & 0.9936 & 0.9796 & 0.9905 & 0.9890 & 0.9816 & 0.9833 & 0.9396 & 1.0000 & 0.9060 & 0.9387 \\
\hline ecoli0 & 0.0000 & 0.3260 & 0.2973 & 0.3463 & 0.3444 & 0.3225 & 0.3787 & 0.6848 & 0.2308 & 0.6173 & 0.6873 \\
\hline glass016vs2 & 0.0000 & 0.3196 & 0.3203 & 0.2686 & 0.3048 & 0.2963 & 0.2102 & 0.1395 & 0.3404 & 0.2001 & 0.2857 \\
\hline ecoli0147vs2356 & 0.0000 & 0.4230 & 0.3014 & 0.4960 & 0.4354 & 0.4435 & 0.5021 & 0.2230 & 0.0500 & 0.4043 & 0.5074 \\
\hline $\mathrm{d} 7$ digit $02456789 \mathrm{vs} 1$ & 0.7748 & 0.5707 & 0.6197 & 0.5226 & 0.5766 & 0.5156 & 0.7308 & 0.5691 & 0.5961 & 0.6746 & 0.7224 \\
\hline ecoli01vs5 & 0.0000 & 0.4138 & 0.2588 & 0.4482 & 0.4103 & 0.4946 & 0.4392 & 0.4140 & 0.2069 & 0.6843 & 0.7811 \\
\hline glass06vs5 & 1.0000 & 0.9057 & 0.9655 & 0.8953 & 0.8857 & 0.9083 & 0.9866 & 0.8654 & 1.0000 & 0.9783 & 0.9474 \\
\hline glass0 & 0.0000 & & & 0.2247 & 0.2473 & 0.2814 & 0.2823 & 0.1747 & 0.2931 & 0.2597 & 0.2768 \\
\hline glass2 & 0.0000 & 0.2477 & 0.2362 & 0.2329 & 0.2478 & 0.2988 & 0.2484 & 0.1131 & 0.3233 & 0.2019 & 0.26 \\
\hline
\end{tabular}




\begin{tabular}{|c|c|c|c|c|c|c|c|c|c|c|c|}
\hline Dataset & Original & SMOTE & ADASYN & STL & sSafe & sRST & sCHC & EUSCHC & AHC & FRB+CHC & FRB \\
\hline ecoli0147vs56 & 0.0000 & 0.5757 & 0.4148 & 0.6288 & 0.6022 & 0.5103 & 0.5164 & 0.6148 & 0.0571 & 0.6762 & 0.7609 \\
\hline cleveland0vs4 & 0.0000 & 0.1539 & 0.1556 & 0.1560 & 0.1263 & 0.1600 & 0.0923 & 0.2621 & 0.0000 & 0.1687 & 0.2030 \\
\hline ecoli0146vs5 & 0.0000 & 0.4280 & 0.1920 & 0.4112 & 0.4356 & 0.4422 & 0.3762 & 0.6993 & 0.3000 & 0.7456 & 0.7758 \\
\hline shuttlec0vsc4 & 0.9490 & 0.9740 & 0.8937 & 0.9749 & 0.9740 & 0.9817 & 0.9724 & 0.9707 & 0.9675 & 0.7964 & 0.8763 \\
\hline yeast1vs7 & 0.0000 & 0.2926 & 0.2870 & 0.2865 & 0.2939 & 0.2738 & 0.3120 & 0.0000 & 0.2861 & 0.3161 & 0.3381 \\
\hline glass4 & 0.8560 & 0.6633 & 0.6565 & 0.6590 & 0.6613 & 0.6463 & 0.8190 & 0.7164 & 0.8471 & 0.7273 & 0.7197 \\
\hline ecoli4 & 0.7500 & 0.6352 & 0.5082 & 0.6354 & 0.6389 & 0.6491 & 0.7931 & 0.7372 & 0.7109 & 0.7356 & 0.6617 \\
\hline page_blocks13vs4 & 0.2270 & 0.2033 & 0.1907 & 0.2010 & 0.2034 & 0.1894 & 0.3563 & 0.0832 & 0.2270 & 0.1816 & 0.1907 \\
\hline abalone918 & 0.0444 & 0.4522 & 0.4172 & 0.4206 & 0.4474 & 0.4570 & 0.5221 & 0.2643 & 0.5303 & 0.5732 & 0.5561 \\
\hline glass016vs5 & 0.6650 & 0.5674 & 0.6592 & 0.5601 & 0.5668 & 0.6551 & 0.7548 & 0.4688 & 0.7273 & 0.7694 & 0.6857 \\
\hline shuttlec2vsc4 & 0.4 & 0.7152 & 0.7152 & 0.7152 & 0.7152 & 0.7288 & 0.6103 & 0.1593 & 0.4 & 0.6126 & 0.7395 \\
\hline yeast1458vs7 & 0 & 0.1318 & 0.1261 & 0.1260 & 0.1323 & 0.1344 & 0.1585 & 0 & 0.1398 & 0.1557 & 0.1187 \\
\hline glass5 & 0.7 & 0.5937 & 0.4551 & 0.5495 & 0.5932 & 0.4838 & 0.6583 & 0.3542 & 0.7 & 0.7533 & 0.8 \\
\hline yeast $2 \mathrm{vs} 8$ & 0.6967 & 0.5972 & 0.2079 & 0.5905 & 0.5989 & 0.5984 & 0.7068 & 0.6967 & 0.6570 & 0.6967 & 0.6967 \\
\hline yeast4 & 0 & 0.2703 & 0.2464 & 0.2648 & 0.2715 & 0.2711 & 0.3076 & 0.0308 & 0.2714 & 0.3533 & 0.3398 \\
\hline yeast $1289 v s 7$ & 0 & 0.1395 & 0.1363 & 0.1357 & 0.1397 & 0.1308 & 0.1851 & 0 & 0.1488 & 0.1967 & 0.1776 \\
\hline yeast5 & 0 & 0.4843 & 0.4611 & 0.4742 & 0.4818 & 0.4751 & 0.5146 & 0.5802 & 0.5012 & 0.4476 & 0.4415 \\
\hline ecoli0137vs26 & 0 & 0.3976 & 0.2400 & 0.4681 & 0.4292 & 0.3636 & 0.4306 & 0.3158 & 0.1 & 0.3465 & 0.3826 \\
\hline yeast6 & 0 & 0.2698 & 0.2014 & 0.2606 & 0.2705 & 0.2670 & 0.3577 & 0 & 0.2756 & 0.3288 & 0.2759 \\
\hline abalone19 & 0 & 0.0408 & 0.0406 & 0.0403 & 0.0409 & 0.0486 & 0.0437 & 0 & 0.0411 & 0.0482 & 0.0445 \\
\hline Mean & 0.2510 & 0.4711 & 0.3917 & 0.4734 & 0.4733 & 0.4693 & 0.5090 & 0.4492 & 0.4039 & 0.5179 & 0.5451 \\
\hline
\end{tabular}


Table 3: SVM: Average AUC of Testing Datasets among Different Sampling Methods.

\begin{tabular}{|c|c|c|c|c|c|c|c|c|c|c|c|}
\hline Dataset & Original & SMOTE & ADASYN & sTL & sSafe & sRST & sCHC & EUSCHC & $\mathbf{A H C}$ & FRB+CHC & FRB \\
\hline ecoli034vs5 & 0.4972 & 0.7069 & 0.5889 & 0.7236 & 0.7047 & 0.6799 & 0.6747 & 0.8111 & 0.5972 & 0.8217 & 0.8472 \\
\hline yeast $2 \mathrm{vs} 4$ & 0.7362 & 0.8924 & 0.8788 & 0.8900 & 0.8931 & 0.8892 & 0.8656 & 0.8804 & 0.8885 & 0.8757 & 0.8424 \\
\hline ecoli067vs35 & 0.5000 & 0.6860 & 0.6625 & 0.7063 & 0.6790 & 0.6700 & 0.6943 & 0.6675 & 0.6200 & 0.7860 & 0.8325 \\
\hline ecoli0234vs5 & 0.4972 & 0.6978 & 0.6140 & 0.7081 & 0.6943 & 0.6820 & 0.7181 & 0.8014 & 0.5917 & 0.8289 & 0.8112 \\
\hline glass $015 \mathrm{vs} 2$ & 0.5000 & 0.7152 & 0.7352 & 0.7284 & 0.7376 & 0.7496 & 0.5905 & 0.4911 & 0.6484 & 0.5530 & 0.575 \\
\hline yeast 0359 vs 78 & 0.6067 & 0.7344 & 0.6936 & 0.7281 & 0.7391 & 0.7334 & 0.7289 & 0.6067 & 0.7371 & 0.6062 & 0.6067 \\
\hline yeast0256vs 3789 & 0.5486 & 0.7960 & 0.7734 & 0.7972 & 0.7965 & 0.7993 & 0.8038 & 0.8064 & 0.7918 & 0.7691 & 0.5761 \\
\hline yeast 02579 vs 368 & 0.8695 & 0.9057 & 0.8610 & 0.9085 & 0.9035 & 0.9071 & 0.9041 & 0.9135 & 0.9052 & 0.9125 & 0.9078 \\
\hline ecoli046vs5 & 0.4973 & 0.6496 & 0.5614 & 0.6488 & 0.6574 & 0.6696 & 0.6395 & 0.7461 & 0.5195 & 0.7880 & 0.8427 \\
\hline ecoli01vs235 & 0.4955 & 0.6606 & 0.5377 & 0.6628 & 0.6598 & 0.6616 & 0.6758 & 0.7423 & 0.5405 & 0.7866 & 0.8659 \\
\hline ecoli0267vs35 & 0.5000 & 0.6073 & 0.5826 & 0.6093 & 0.6020 & 0.6113 & 0.6405 & 0.7035 & 0.5450 & 0.8176 & 0.8483 \\
\hline glass04vs5 & 1.0000 & 0.9754 & 0.9754 & 0.9728 & 0.9842 & 0.9830 & 0.9988 & 0.9570 & 1.0000 & 0.9732 & 0.9938 \\
\hline ecoli0346vs5 & 0.4973 & 0.6974 & 0.6115 & 0.7421 & 0.7124 & 0.7127 & 0.7170 & 0.7878 & 0.6169 & 0.8459 & 0.8656 \\
\hline ecoli0347vs56 & 0.5000 & 0.7569 & 0.7028 & 0.7594 & 0.7444 & 0.7294 & 0.7511 & 0.8071 & 0.5579 & 0.7888 & 0.8310 \\
\hline yeast $05679 \mathrm{vs} 4$ & 0.5000 & 0.7869 & 0.7902 & 0.7862 & 0.7861 & 0.7797 & 0.7934 & 0.7860 & 0.7754 & 0.7899 & 0.7786 \\
\hline vowel0 & 1.0000 & 0.9993 & 0.9978 & 0.9990 & 0.9988 & 0.9981 & 0.9982 & 0.9933 & 1.0000 & 0.9892 & 0.9933 \\
\hline ecoli067vs5 & 0.5000 & 0.6103 & 0.6100 & 0.6155 & 0.6175 & 0.6106 & 0.6245 & 0.8000 & 0.5725 & 0.8125 & 0.845 \\
\hline glass016vs2 & 0.5000 & 0.7529 & 0.7529 & 0.7106 & 0.7464 & 0.7322 & 0.6239 & 0.5733 & 0.7517 & 0.6114 & 0.6552 \\
\hline ecoli0147vs2356 & 0.4984 & 0.6509 & 0.6154 & 0.6920 & 0.6580 & 0.6629 & 0.6891 & 0.6504 & 0.5102 & 0.8054 & 0.8441 \\
\hline led7digit02456789vs1 & 0.8788 & 0.8819 & 0.8867 & 0.8799 & 0.8856 & 0.8650 & 0.8946 & 0.9055 & 0.8600 & 0.8844 & 0.8921 \\
\hline ecoli01vs5 & 0.4977 & 0.6602 & 0.5864 & 0.6786 & 0.6566 & 0.6875 & 0.6659 & 0.7091 & 0.5727 & 0.8159 & 0.8432 \\
\hline glass06vs5 & 1.0000 & 0.9774 & 0.9950 & 0.9574 & 0.9629 & 0.9436 & 0.9895 & 0.9397 & 1.0000 & 0.9840 & 0.95 \\
\hline glass0146vs2 & 0.5000 & 0.6823 & 0.6849 & 0.6594 & 0.6821 & 0.7142 & 0.6717 & 0.5519 & 0.7153 & 0.6336 & 0.6808 \\
\hline glass2 & 0.5000 & 0.7132 & 0.6981 & 0.6938 & 0.7127 & 0.7607 & 0.6648 & 0.5248 & 0.7868 & 0.6078 & 0.6875 \\
\hline
\end{tabular}




\begin{tabular}{|c|c|c|c|c|c|c|c|c|c|c|c|}
\hline Dataset & Original & SMOTE & ADASYN & sTL & sSafe & sRST & sCHC & EUSCHC & AHC & FRB+CHC & FRB \\
\hline ecoli0147vs56 & 0.5000 & 0.7160 & 0.6352 & 0.7460 & 0.7335 & 0.7053 & 0.6905 & 0.7722 & 0.5167 & 0.8578 & 0.9171 \\
\hline cleveland0vs4 & 0.4969 & 0.5622 & 0.5575 & 0.5526 & 0.5321 & 0.5421 & 0.5210 & 0.5991 & 0.4811 & 0.5857 & 0.6034 \\
\hline ecoli0146vs5 & 0.4981 & 0.6440 & 0.5654 & 0.6394 & 0.6467 & 0.6558 & 0.6260 & 0.7731 & 0.5962 & 0.8371 & 0.8442 \\
\hline shuttlec0vsc4 & 0.9515 & 0.9747 & 0.9872 & 0.9755 & 0.9747 & 0.9845 & 0.9731 & 0.9715 & 0.9749 & 0.9812 & 0.9897 \\
\hline yeast1vs7 & 0.5000 & 0.7583 & 0.7744 & 0.7632 & 0.7602 & 0.7500 & 0.6777 & 0.5000 & 0.7261 & 0.6932 & 0.7579 \\
\hline glass4 & 0.9092 & 0.9148 & 0.9176 & 0.9113 & 0.9143 & 0.9163 & 0.9333 & 0.9251 & 0.9350 & 0.9230 & 0.8942 \\
\hline ecoli4 & 0.8000 & 0.9101 & 0.9149 & 0.9143 & 0.9171 & 0.9426 & 0.9244 & 0.9528 & 0.9279 & 0.9368 & 0.9231 \\
\hline page_blocks13vs4 & 0.5700 & 0.7528 & 0.7320 & 0.7493 & 0.7531 & 0.7298 & 0.6847 & 0.5609 & 0.5689 & 0.7141 & 0.732 \\
\hline abalone918 & 0.5125 & 0.8961 & 0.8860 & 0.8863 & 0.8939 & 0.8916 & 0.8745 & 0.5792 & 0.9144 & 0.8597 & 0.83 \\
\hline glass016vs5 & 0.8443 & 0.8856 & 0.9186 & 0.8791 & 0.8853 & 0.9221 & 0.8979 & 0.8071 & 0.8943 & 0.9186 & 0.8886 \\
\hline shuttlec $2 \mathrm{vsc} 4$ & 0.7 & 0.9548 & 0.9548 & 0.9548 & 0.9548 & 0.9590 & 0.9440 & 0.6957 & 0.7 & 0.9493 & 0.9632 \\
\hline yeast1458vs7 & 0.5 & 0.6427 & 0.6373 & 0.6396 & 0.6444 & 0.6539 & 0.6638 & 0.5 & 0.6546 & 0.5958 & 0.5954 \\
\hline glass5 & 0.8451 & 0.8760 & 0.8256 & 0.8807 & 0.8845 & 0.8515 & 0.8515 & 0.8768 & 0.8451 & 0.8967 & 0.8927 \\
\hline yeast $2 \mathrm{vs} 8$ & 0.7739 & 0.7628 & 0.7242 & 0.7614 & 0.7633 & 0.7770 & 0.7852 & 0.7739 & 0.8381 & 0.7739 & 0.7739 \\
\hline yeast4 & 0.5 & 0.8156 & 0.8102 & 0.8227 & 0.8160 & 0.8124 & 0.8177 & 0.5093 & 0.8127 & 0.7991 & 0.7663 \\
\hline yeast $1289 \mathrm{vs} 7$ & 0.5 & 0.7141 & 0.7145 & 0.7133 & 0.7109 & 0.6968 & 0.7201 & 0.5 & 0.7202 & 0.6990 & 0.7453 \\
\hline yeast5 & 0.5 & 0.9668 & 0.9635 & 0.9655 & 0.9665 & 0.9655 & 0.9683 & 0.7976 & 0.9691 & 0.9621 & 0.9611 \\
\hline ecoli0137vs26 & 0.5 & 0.7118 & 0.5927 & 0.7390 & 0.7413 & 0.6909 & 0.7294 & 0.6427 & 0.5463 & 0.6655 & 0.6945 \\
\hline yeast6 & 0.5 & 0.8742 & 0.8597 & 0.8716 & 0.8744 & 0.8736 & 0.8735 & 0.5 & 0.8761 & 0.8880 & 0.8880 \\
\hline abalone19 & 0.5 & 0.7177 & 0.7170 & 0.7163 & 0.7180 & 0.7715 & 0.7166 & 0.5 & 0.6881 & 0.7016 & 0.7063 \\
\hline Mean & 0.6141 & 0.7784 & 0.7519 & 0.7805 & 0.7795 & 0.7801 & 0.7703 & 0.7248 & 0.7339 & 0.8020 & 0.8133 \\
\hline
\end{tabular}


Table 4: Friedman Rankings of AUC and F-measure.

\begin{tabular}{|c|c|c|}
\hline Preprocessing Method & AUC & F-measure \\
\hline Original & 8.841 & 7.568 \\
SMOTE & 4.636 & 5.341 \\
ADASYN & 6.159 & 7.341 \\
sTL & 4.864 & 5.636 \\
sSafe & 4.624 & 4.932 \\
SRST & 4.659 & 5.318 \\
SCHC & 4.886 & $\mathbf{3 . 4 7 7}$ \\
EUSCHC & 6 & 5.659 \\
AHC & 5.773 & 5.5 \\
FRB+CHC & $\mathbf{4 . 4 5 5}$ & 3.886 \\
\hline
\end{tabular}

Although the hybrid sampling methods can get better results, the main drawback of them is that the size of training set is expanded greatly. If IR of the dataset is large, the size of the re-sampled training set can be nearly double of the original one. This drawback may increase the computational time and complexity of the learning model. Table 5 shows the over-sampling rates of different methods on each dataset and the mean rate of each method. A negative value means the size of re-sampled training set is smaller than that of original one. A value greater than $100 \%$ means the size of re-sampled training set is more than 2 times of the original set. Both $\mathrm{sCHC}$ and $\mathrm{FRB}+\mathrm{CHC}$ shrink most of the dataset while the oversampling rates of the other methods are similar. This shows that both sCHC and $\mathrm{FRB}+\mathrm{CHC}$ can use less training samples to achieve high performance. Table 6 
shows the details of the re-sampled training sets after applying FRB+CHC. It reveals the decrease rate of the majority class, the increase rate of the minority class, and the updated IR. The IR values of re-sampled training sets are not always equal to one because $\mathrm{CHC}$ makes use of a fitness function to select a subset of samples. The range of IR is between 0.9 and 1.5 .

Table 7 shows the increase rate of the number of support vectors used to form the classification model. The number of support vectors can reflect the complexity of the classification model formed by SVM. When the number of support vectors is smaller, the classification model is more easily applied. Some negative values can be found since the number of support vectors for the re-sampled dataset is less than that of the original dataset. Both $\mathrm{sCHC}$ and $\mathrm{FRB}+\mathrm{CHC}$ have the smallest increase rate of the number of support vectors on average. The average number of support vectors are only increased by around 0.776 times and 0.948 times of the original datasets; while most of the other methods have the number increased by over 2 times.

The results of sCHC and $\mathrm{FRB}+\mathrm{CHC}$ are similar from the above tables. To show the difference of these two methods, Fig. 5 reveal the average $A U C$ results obtained from the training and testing sets (sorted by the nonlinearity of the $1 \mathrm{NN}$ classifier.) The x-axis shows the selected 44 datasets. The solid lines in the figures represent the average $A U C$ results for the testing set; the dashed lines represent the average $A U C$ results for the training set. $\mathrm{FRB}+\mathrm{CHC}$ shows the advantage 
Table 5: Over-sampling Rate (\%) of Training Sets among Different Sampling Methods.

\begin{tabular}{|c|c|c|c|c|c|c|c|c|}
\hline Dataset & SMOTE & ADASYN & STL & SSafe & SRST & SCHC & AHC & FRB+CHC \\
\hline ecoli0347vs56 & 80.53 & 80 & 77.63 & 80.53 & 100.77 & -5.60 & 80 & -3.06 \\
\hline yeast $2 \mathrm{vs} 4$ & 80.16 & 80.16 & 76.85 & 80.16 & 80.16 & -3.13 & 80.16 & -3.90 \\
\hline ecoli067vs35 & 80.18 & 80.18 & 77.14 & 80.18 & 94.13 & -5.19 & 80.18 & -4.16 \\
\hline ecoli0234vs5 & 80.20 & 80.20 & 77.10 & 80.20 & 89.36 & -4.46 & 80.20 & -5.50 \\
\hline glass015vs2 & 80.23 & 80.23 & 70.79 & 80.23 & 80.23 & -2.06 & 80.23 & -2.28 \\
\hline yeast 0359 vs 78 & 80.24 & 80.24 & 71.49 & 80.24 & 80.34 & -4.90 & 80.24 & -2.14 \\
\hline yeast 0256 vs 3789 & 80.28 & 80.28 & 74.25 & 80.28 & 80.73 & -5.70 & 80.28 & -0.92 \\
\hline yeast 02579 vs 368 & 8 & 80.28 & 76.97 & 80.28 & 80.28 & -3.46 & 80.28 & -2.52 \\
\hline ecoli046vs5 & 80.30 & 80.30 & 77.09 & 80.30 & 112.06 & -3.99 & 80.30 & -2.72 \\
\hline $47 v s 2356$ & 84.01 & 80.33 & 80.02 & 84.01 & 119.57 & -4.05 & 80.33 & -1.50 \\
\hline ecoli0267vs35 & 80.36 & 80.36 & 76.56 & 80.36 & 94.65 & -4.03 & 80.36 & -2.62 \\
\hline & 44 & 80.44 & & 80.44 & 96.76 & -4.40 & 80.44 & -2.91 \\
\hline ecoli & 80.20 & 80.49 & 77.72 & 80.20 & 86.63 & -4.43 & 80.49 & -2.73 \\
\hline ecol & 80.39 & 80.54 & 78.18 & 80.39 & & -3.54 & 80.54 & -3.12 \\
\hline yeast 05679 vs 4 & 80.68 & 80.68 & 74.48 & 80.68 & 80.68 & -5.71 & 80.68 & -2.48 \\
\hline 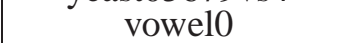 & 81.78 & 81.78 & 81.78 & 81.78 & 84.56 & -4.82 & 81.78 & -4.29 \\
\hline ecoli & 81.82 & 81.82 & 75.68 & 81.82 & 87.27 & -4.78 & 81.82 & -1.68 \\
\hline glass & 82.29 & 82.29 & & 82.29 & 82.29 & -0.78 & 82.29 & -2.11 \\
\hline $7 \mathrm{vs} 26$ & 90.48 & 82.74 & 87.65 & 90.48 & 169.85 & -1.33 & 82.74 & 0.28 \\
\hline led7digit02456789vs1 & 83.30 & 83.30 & 78.39 & 83.30 & 94.02 & -3.93 & 83.30 & -7.90 \\
\hline ecoli0147vs56 & 83.97 & 83.33 & 79.91 & 83.97 & 137.03 & -3.39 & 83.33 & -1.73 \\
\hline & 83.34 & 83.34 & & 83.34 & 91.22 & -2.71 & 83.34 & \\
\hline glass & 83.41 & 83.41 & 74.63 & 83.41 & 83.41 & -0.94 & 83.41 & -1.40 \\
\hline gla & 84.11 & 84.11 & & 84.11 & 84.11 & -2.07 & 84.11 & -0.42 \\
\hline ecolio & 89.30 & 84.94 & 86.00 & 89.30 & 139.65 & -3.34 & 84.94 & -2.73 \\
\hline clev & 8 & 84.97 & & 84.97 & 205.49 & -3.27 & 84.97 & -1.13 \\
\hline & 92.75 & 85.71 & 51 & 92.75 & 186.47 & -3.58 & 85.71 & -1.22 \\
\hline shu & 86 & & & 86.55 & 136.58 & -3.32 & 86.55 & -3.17 \\
\hline yeast & 91.81 & 86.93 & & 91.81 & 91.81 & -1.13 & 86.93 & -2.51 \\
\hline & & & & & 112.84 & -2.68 & 87.85 & -0.95 \\
\hline $\mathrm{ec}$ & 88.10 & 88.10 & 86.46 & 88.10 & 88.39 & -1.59 & 88.10 & -1.40 \\
\hline page_blo & 88 & 88.14 & & 88.14 & 157.10 & -2.88 & 88.14 & -0.86 \\
\hline & & & & 88.58 & 88.58 & -1.72 & 88.58 & -1.44 \\
\hline & 9 & 90 & & 22 & & -2.84 & 90.22 & -1.03 \\
\hline shutt & 90.70 & 90.70 & 89.92 & 90.70 & 113.19 & -3.86 & 90.70 & -3.43 \\
\hline ye & 92.3 & 91.34 & 88.44 & 92.32 & 92.32 & -2.50 & 91.34 & 235 \\
\hline gla & 91.59 & 91.59 & 89.37 & 91.59 & 92.76 & -1.58 & 91.59 & 0.12 \\
\hline yea & 91.70 & 91.70 & 89.83 & 91.70 & 98.44 & -1.86 & 91.70 & -0.46 \\
\hline ye & 93.13 & 93.13 & 90.09 & 93.13 & 93.13 & -1.69 & 93.13 & -1.16 \\
\hline yeas & 89. & 93.66 & 85.04 & 89.62 & 89.62 & -3.39 & 93.66 & \\
\hline ye & 94.0 & 94.07 & 92.62 & 94.07 & 94.07 & -1.66 & 94.07 & -2.09 \\
\hline ecoli01vs 235 & 82.59 & 95.02 & 78.95 & 82.59 & 106.04 & -4.04 & 95.02 & -0.59 \\
\hline ye & 95.28 & 95.28 & 93.36 & 95.28 & 95.28 & -2.54 & 95.28 & -1.88 \\
\hline abal & 98.47 & 98.47 & 97.32 & 98.47 & 98.47 & 1.04 & 98.47 & -0.31 \\
\hline Mean & 85.70 & 85.40 & 81.94 & 85.70 & 103.47 & -3.13 & 85.40 & -2.17 \\
\hline
\end{tabular}


Table 6: The Details of the Re-sampled Datasets After FRB+CHC.

\begin{tabular}{|c|c|c|c|}
\hline Dataset & Decrease Rate of & Increase Rate of & \\
& & & Updated IR \\
\hline ecoli034vs5 & Majority Class & Minority Class & \\
yeast2vs4 & 0.474 & 3.700 & 1.010 \\
ecoli067vs35 & 0.469 & 3.865 & 0.995 \\
ecoli0234vs5 & 0.400 & 4.224 & 0.874 \\
glass015vs2 & 0.405 & 3.463 & 1.048 \\
yeast0359vs78 & 0.418 & 2.989 & 1.369 \\
yeast0256vs3789 & 0.452 & 3.995 & 1.064 \\
yeast02579vs368 & 0.456 & 4.005 & 1.002 \\
ecoli046vs5 & 0.469 & 3.841 & 1.028 \\
ecoli01vs235 & 0.445 & 3.900 & 1.001 \\
ecoli0267vs35 & 0.500 & 3.939 & 1.030 \\
glass04vs5 & 0.464 & 3.756 & 0.969 \\
ecoli0346vs5 & 0.476 & 4.325 & 0.934 \\
ecoli0347vs56 & 0.452 & 4.188 & 0.935 \\
yeast05679vs4 & 0.441 & 3.940 & 1.032 \\
vowe10 & 0.514 & 3.938 & 1.064 \\
ecoli067vs5 & 0.461 & 4.619 & 0.863 \\
glass016vs2 & 0.393 & 4.025 & 1.076 \\
ecoli0147vs2356 & 0.424 & 3.635 & 1.357 \\
led7digit02456789vs1 & 0.499 & 4.651 & 1.084 \\
ecoli01vs5 & 0.470 & 4.603 & 0.983 \\
glass06vs5 & 0.482 & 4.975 & 0.978 \\
glass0146vs2 & 0.392 & 5.339 & 0.910 \\
glass2 & 0.449 & 4.568 & 1.212 \\
ecoli0147vs56 & 0.475 & 4.826 & 1.101 \\
cleveland0vs4 & 0.442 & 5.510 & 0.992 \\
ecoli0146vs5 & 0.470 & 6.049 & 0.984 \\
shuttlec0vsc4 & 0.507 & 5.550 & 1.053 \\
yeast1vs7 & 0.420 & 6.530 & 0.908 \\
glass4 & 0.489 & 5.525 & 1.277 \\
ecoli4 & 0.463 & 6.651 & 1.038 \\
page_blocks13vs4 & 0.480 & 7.300 & 1.024 \\
abalone918 & 0.478 & 7.236 & 1.003 \\
glass016vs5 & 0.486 & 7.658 & 0.994 \\
shuttlec2vsc4 & 0.514 & 9.296 & 0.974 \\
yeast1458vs7 & 0.391 & 7.900 & 1.936 \\
glass5 & 0.478 & 10.321 & 1.052 \\
yeast2vs8 & 0.452 & 10.500 & 1.105 \\
yeast4 & 0.468 & 12.397 & 1.117 \\
yeast1289vs7 & 0.418 & 11.967 & 1.083 \\
yeast5 & 0.480 & 14.768 & 1.023 \\
ecoli0137vs26 & 0.467 & 1.633 & 1.067 \\
yeast6 & 0.451 & 61.247 & \\
abalone19 & 0.487 & & \\
\hline
\end{tabular}


Table 7: The Increase Rate of Number of Support Vectors of the Classification Model formed by

SVM.

\begin{tabular}{|c|c|c|c|c|c|c|c|c|}
\hline Dataset & SMOTE & ADASYN & sTL & SSafe & SRST & sCHC & AHC & FRB+CHC \\
\hline ecoli0347vs56 & 0.245 & 0.529 & 0.418 & 0.143 & 0.476 & -0.117 & 0.178 & 0.026 \\
\hline yeast $2 \mathrm{vs} 4$ & 2.662 & 4.354 & 2.507 & 2.981 & 2.698 & 1.180 & 2.377 & 1.000 \\
\hline ecoli067vs35 & 0.252 & 0.506 & 0.468 & 0.249 & 0.465 & -0.103 & 0.215 & 0.021 \\
\hline ecoli0234vs5 & 0.272 & 0.538 & 0.472 & 0.122 & 0.473 & -0.097 & 0.197 & 0.011 \\
\hline glass $015 \mathrm{vs} 2$ & 3.731 & 3.687 & 3.193 & 3.996 & 3.583 & 1.705 & 3.310 & 1.279 \\
\hline yeast0359vs 78 & 1.384 & 1.676 & 1.149 & 1.434 & 1.418 & 0.251 & 1.361 & 0.093 \\
\hline yeast $0256 \mathrm{vs} 3789$ & 3.555 & 5.151 & 3.240 & 3.669 & 3.577 & 1.414 & 3.416 & 0.990 \\
\hline yeast 02579 vs 368 & 2.142 & 5.240 & 1.919 & 2.251 & 2.188 & 0.783 & 1.990 & 0.521 \\
\hline ecoli046vs5 & 0.263 & 0.477 & 0.460 & 0.125 & 0.490 & -0.100 & 0.177 & 0.031 \\
\hline ecoli01 & 0.229 & 0.566 & 0.435 & 0.170 & 0.494 & -0.103 & 0.186 & 0.053 \\
\hline ecoli0267vs35 & 0.279 & 0.521 & 0.490 & 0.234 & 0.467 & -0.092 & 0.209 & 0.040 \\
\hline & 1.839 & 1.477 & 1.467 & 4.104 & 1.242 & -0.043 & 0.122 & 0.306 \\
\hline ecoli034vs5 & 0.269 & 0.526 & 0.456 & 0.135 & 0.477 & -0.108 & 0.163 & 0.029 \\
\hline ecoli( & 0.286 & 0.424 & 0.488 & 0.140 & 0.497 & -0.094 & 0.150 & 0.031 \\
\hline yeast $05679 v s 4$ & 3.500 & 4.147 & 3.113 & 3.561 & 3.575 & 1.401 & 3.474 & 1.469 \\
\hline & & 1.333 & 0.752 & 2.311 & 0.972 & -0.027 & 0.448 & 1.631 \\
\hline ecoli067vs5 & 0.227 & 0.440 & 0.391 & 0.212 & 0.438 & -0.109 & 0.144 & 0.055 \\
\hline glass016vs2 & 3.868 & 3.927 & 3.296 & 4.209 & 3.660 & 1.840 & 3.291 & 1.530 \\
\hline ecoli0137vs26 & 0.175 & 0.404 & 0.338 & 0.089 & 0.439 & -0.191 & 0.160 & -0.146 \\
\hline led7digit02456789vs1 & 3.332 & 3.322 & 2.864 & 4.139 & 2.838 & 0.360 & 2.6 & 0.563 \\
\hline ecoli0147vs56 & 0.218 & 0.487 & 0.391 & 0.121 & 0.522 & -0.145 & 0.147 & 0.031 \\
\hline glas & 1.494 & 1.272 & 1.124 & 2.924 & 1.204 & 0.021 & 0.077 & 0.250 \\
\hline glass0 & 3.967 & 4.008 & 3.396 & 4.271 & 3.725 & 1.915 & 3.391 & 1.643 \\
\hline & & 3.893 & & 4.036 & 3.671 & & 3.444 & 1.712 \\
\hline ecolio & 0.189 & 0.379 & 0.361 & 0.105 & 0.394 & -0.155 & 0.137 & 0.014 \\
\hline cleve & 0.540 & 0.741 & 0.712 & 0.247 & 0.505 & -0.077 & 0.3 & 0.008 \\
\hline & & 0.494 & 0.309 & 0.082 & 0.417 & -0.128 & 0. & 0.032 \\
\hline shut & & 2.557 & 64 & 0.001 & 0.450 & & & 1.326 \\
\hline yeas & 3.1 & 6.093 & 2.947 & 3.233 & 3.100 & 1.064 & 5.3 & 0.966 \\
\hline & & 1.873 & & & & & & 0.344 \\
\hline & & 2.998 & 2.020 & 2.668 & 2.394 & 0. & 1. & 1.012 \\
\hline page_blo & 0.8 & 1.042 & 0.863 & 0.057 & 1.198 & & 0.523 & 0.110 \\
\hline abalo & & 9.046 & 8.058 & 10.025 & 8.077 & & 6.7 & 4.309 \\
\hline gl & & 1.760 & 1.440 & & 1.846 & & 0. & 0.512 \\
\hline shutt & 0.6 & 1.428 & 1.388 & 0.1 & 1.310 & & & 0.422 \\
\hline & & 2.993 & & & 5.087 & & & 1.491 \\
\hline glass5 & 2.307 & 2.169 & 1.884 & 4.623 & 2.151 & 0.249 & 0.524 & 0.574 \\
\hline yeast $2 \mathrm{vs} 8$ & 4.905 & 8.609 & 4.920 & & 5.169 & & 4.436 & 1.184 \\
\hline ye & & 3.547 & 2.902 & 3.379 & 3.173 & & 2.966 & 0.919 \\
\hline yeast1vs7 & 5. & 4.672 & 4.870 & 5.523 & 5.3 & 2.673 & 4.0 & 2.422 \\
\hline ye & & 3.458 & 2.617 & 3.676 & 3.265 & 1.578 & 2.930 & 2.007 \\
\hline ecoli01vs235 & 0.2 & 0.2 & 0.463 & 0.1 & 0.484 & -0. & 0.0 & 0.041 \\
\hline ye & & 9.553 & & 7.216 & 6.900 & & 6.479 & 2.632 \\
\hline abalo & 13.156 & 13.209 & 12.829 & 14.221 & 12.973 & 5.535 & 10.986 & 8.226 \\
\hline Mean & 2.351 & 2.859 & 2.198 & 2.708 & 2.403 & 0.776 & 1.887 & 0.948 \\
\hline
\end{tabular}


on relaxing the over-fitting problem since the performances on training set and testing set are similar.

Fig. 6 and 7 show an example of the distribution of the positive samples and negative samples after the re-sampling of $\mathrm{FRB}+\mathrm{CHC}$ and $\mathrm{sCHC}$ respectively. The circle dots correspond to the samples of the majority class. The square dots correspond to the samples of the original minority class. The triangle dots correspond to the synthetic samples. Fig. 7 show that the synthetic samples are generated densely around some of the original minority samples. On the contrary, the synthetic samples in Fig. 6 are distributed more evenly in the area of the original minority samples. Therefore, sCHC runs into the over-fitting problem more easily.

Figs. 8 and 9 show the overall results in terms of $F-$ measure and $A U C$ for different classifiers respectively. Only a small difference of the results for 1NN among all the preprocessing methods is revealed. FRB+CHC obtains the highest value of $A U C$ for both $\mathrm{C} 4.5$ and $1 \mathrm{NN}$. An improvement by $\mathrm{FRB}+\mathrm{CHC}$ in terms of $F-$ measure is shown. In addition, a robust behavior of $\mathrm{FRB}+\mathrm{CHC}$ is shown when the results of the three classifiers only have a small difference. Most of the preprocessing methods can perform better than the original datasets in terms of the average values of $F-$ measure and $A U C$. This confirms that preprocessing is an important step to deal with imbalanced datasets. 


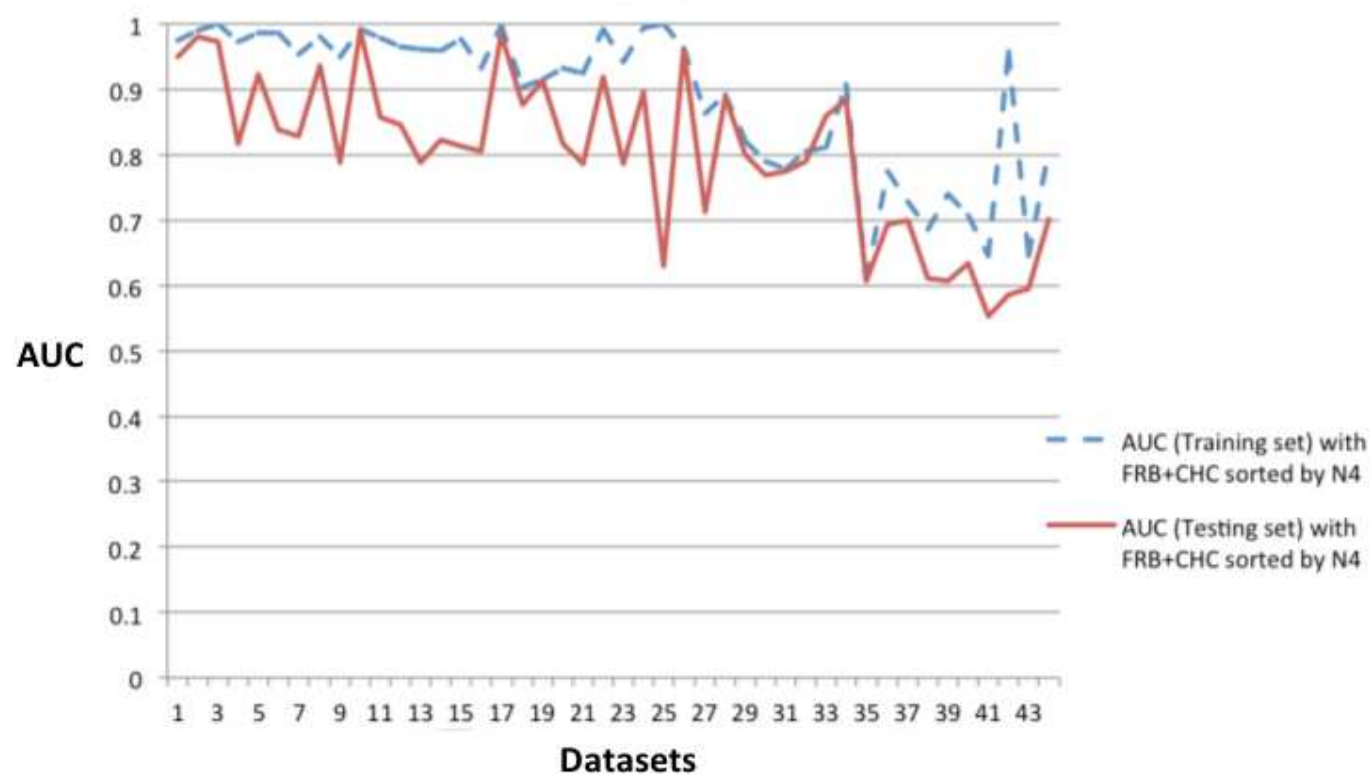

(a) AUC results with $\mathrm{FRB}+\mathrm{CHC}$

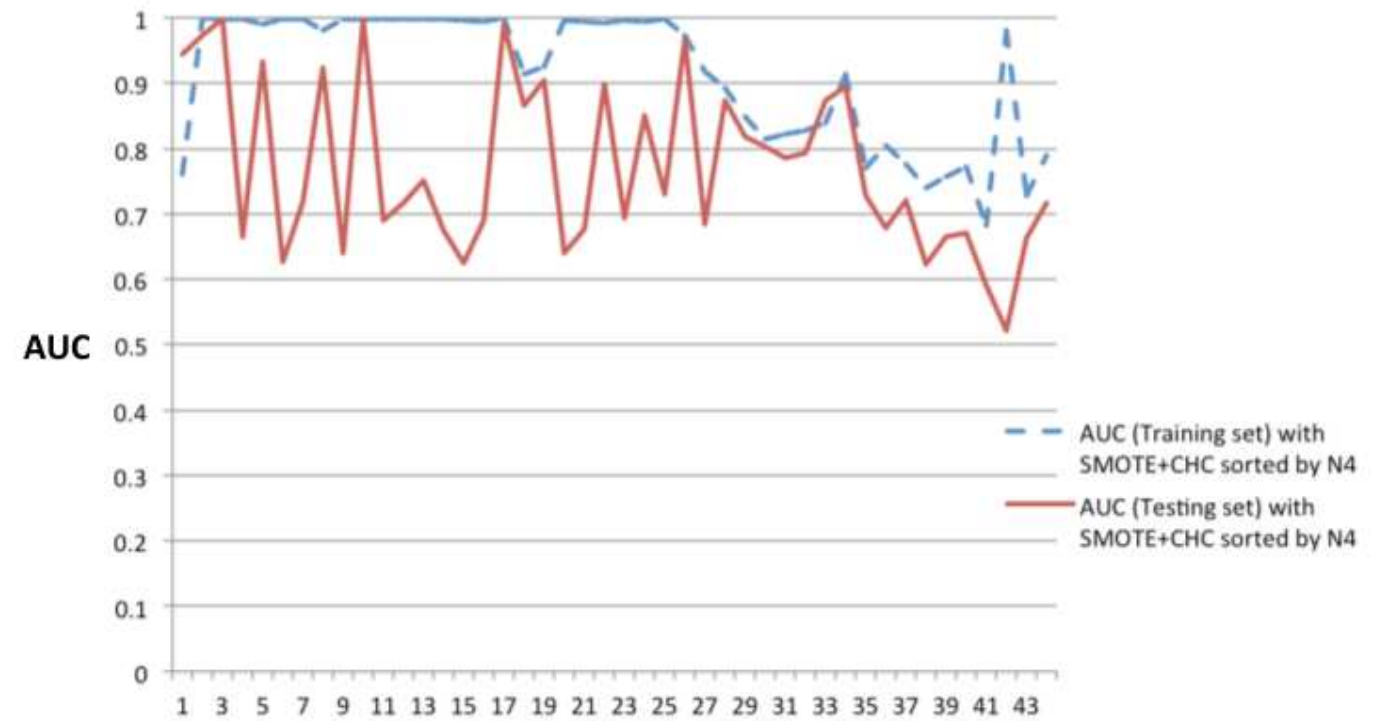

Datasets

(b) AUC results with $\mathrm{sCHC}$

Figure 5: Average AUC results obtained from training and testing sets. 


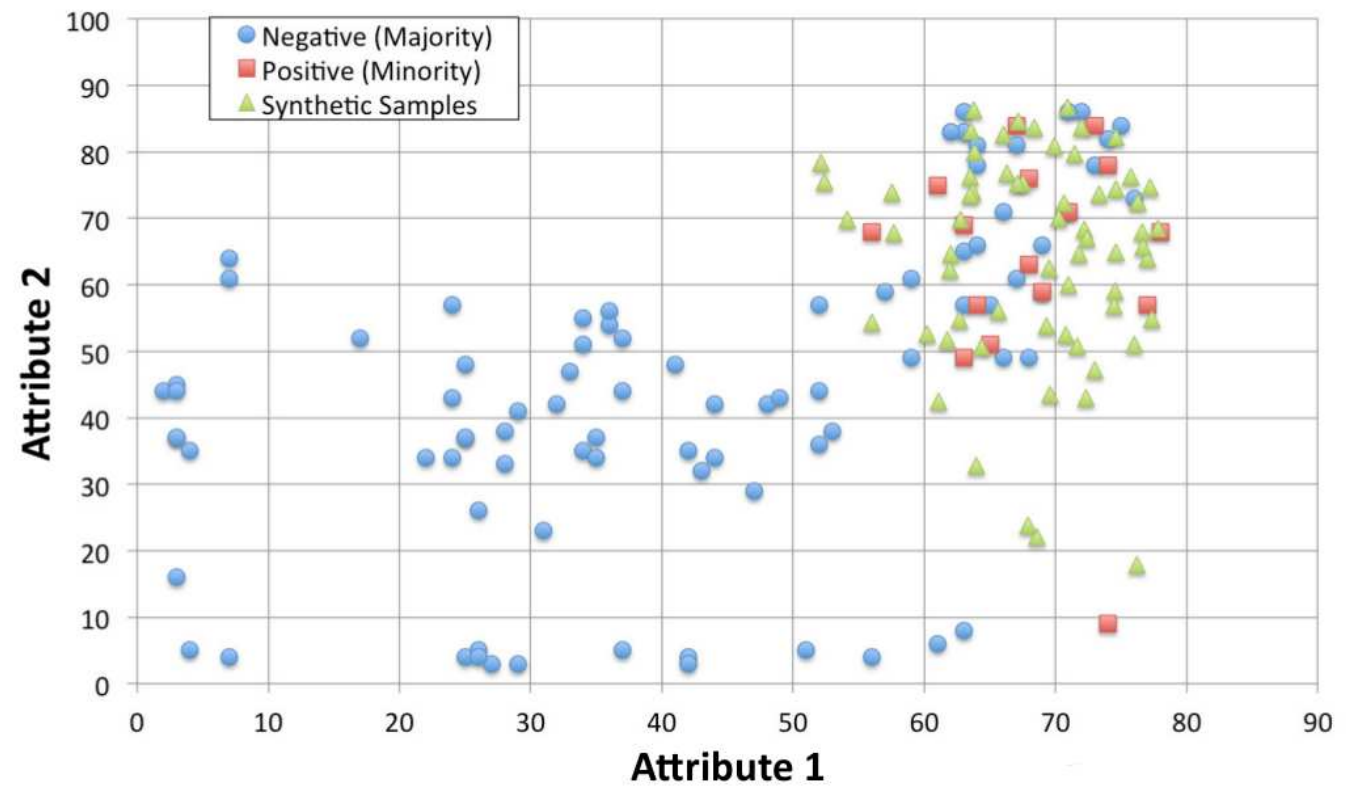

Figure 6: Distribution of the samples after the implementation of FRB+CHC.

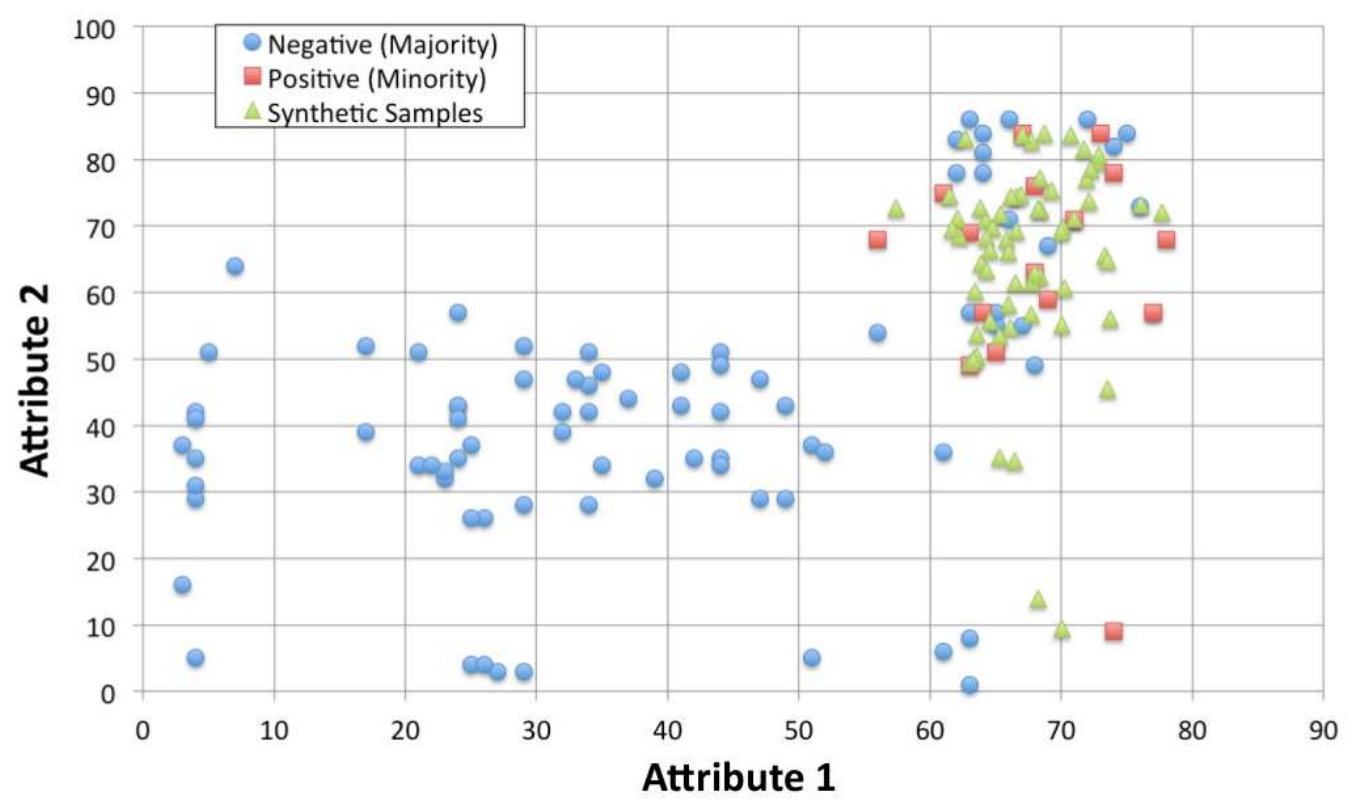

Figure 7: Distribution of the samples after the implementation of sCHC. 


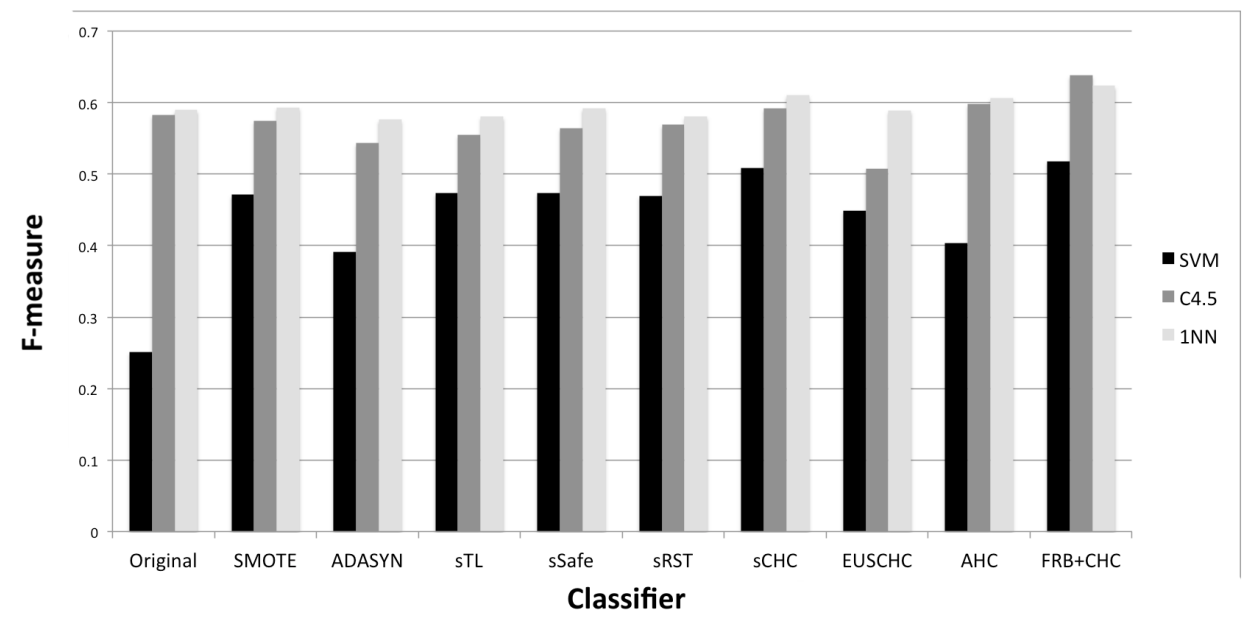

Figure 8: Average F-measure for different classifiers.

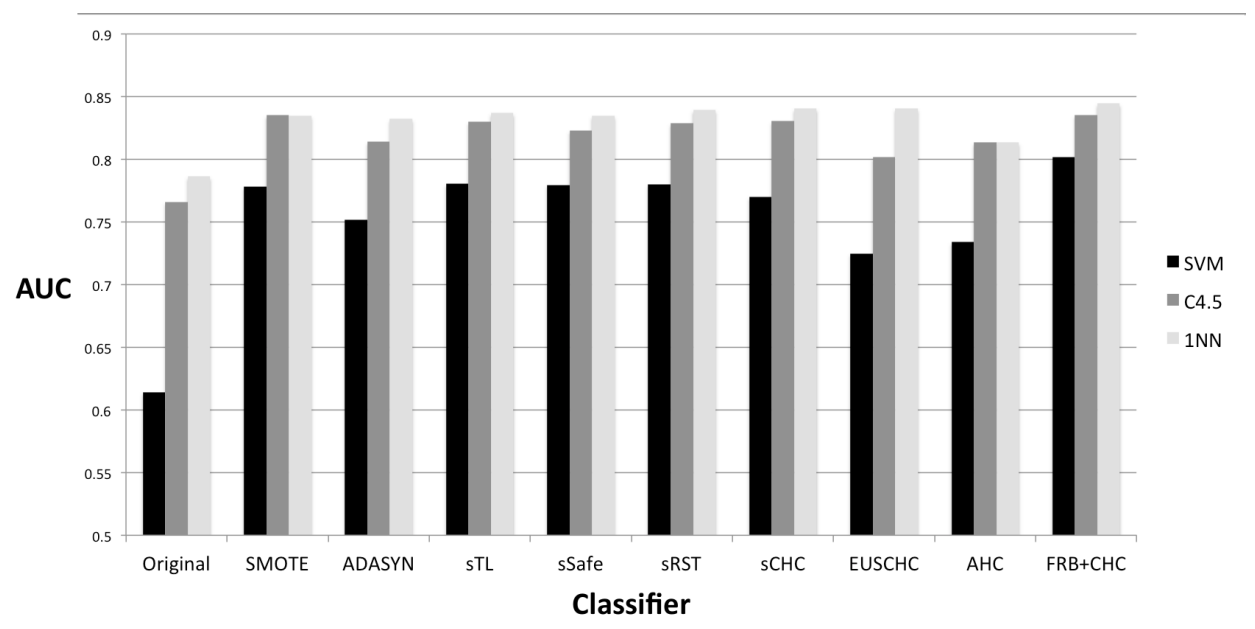

Figure 9: Average AUC for different classifiers. 


\section{Conclusion}

A hybrid re-sampling method developed based on both over-sampling and under-sampling has been proposed. The new synthetic samples of the minority class are generated based on fuzzy logic. To minimize the size of datasets, $\mathrm{CHC}$ has been employed over the new samples and the majority samples as a cleaning method to the over-sampled training set.

The proposed sampling method $(\mathrm{FRB}+\mathrm{CHC})$ is compared to SMOTE, ADASYN, sTL, sSafe, sRST, sCHC, EUSCHC, and AHC on 44 datasets. To evaluate the performance of these nine sampling methods, the same SVM classifier has been used to obtain the experimental results. It is shown that FRB and FRB+CHC outperforms the other sampling methods on both $F-$ measure and $A U C$. FRB shows its advantage to act as an over-sampling method. If data size is not a consideration, FRB is a better choice of pre-processing method.

$\mathrm{FRB}+\mathrm{CHC}$ obtains the best ranking by means of $A U C . \mathrm{FRB}+\mathrm{CHC}$ and $\mathrm{sCHC}$ have similar performance in $F-$ measure, which indicates that $\mathrm{CHC}$ is a good choice of data cleaning method. The $A U C$ results of SMOTE, sTL, sSafe, sRST, and sCHC are similar since all of them use SMOTE to perform over-sampling. To show the advantages of the proposed method, the over-sampling rate and the number of support vectors formed from SVM for different methods are also compared. In addition, the $\mathrm{C} 4.5$ and $1 \mathrm{NN}$ classifiers are used and $\mathrm{FRB}+\mathrm{CHC}$ shows a robust 
behavior among different classifiers. FRB+CHC achieves good results under the above criteria, which reflects that $\mathrm{FRB}+\mathrm{CHC}$ achieves a good balance between accuracy and over-sampling rate. It also has a low impact to the complexity of the learning model. The major reason is that $\mathrm{CHC}$ only selects the samples to increase the performance of the datasets, but not considering the locations of the samples. Therefore, the most representative samples are selected to form the training sets.

\section{Acknowledgment}

The work described in this paper was substantially supported by a grant from The Hong Kong Polytechnic University (Project No. RPKP, RUG7 and G-YN19).

[1] Alcalá-Fdez, J., Fernandez, A., Luengo, J., Derrac, J., García, S., Sánchez, L., and Herrera, F. (2011). Keel data-mining software tool: data set repository, integration of algorithms and experimental analysis framework. Journal of Multiple-Valued Logic and Soft Computing, 17(2-3):255-287.

[2] Asuncion, A. and Newman, D. (2007). Uci machine learning repository. http://www.ics.uci.edu/ mlearn/MLRepository.html.

[3] Batista, G., Prati, R., and Monard, M. (2004). A study of the behavior of several methods for balancing machine learning training data. SIGKDD Explorations, 6(1):20-29. 
[4] Bunkhumpornpat, C., Sinapiromsaran, K., and Lursinsap, C. (2009). Safelevel-smote: Safe-level-synthetic minority over-sampling technique for handling the class imbalanced problem. In Proceedings of the 13th Pacific-Asia Conference on Advances in Knowledge Discovery and Data Mining, PAKDD '09, pages 475-482, Berlin, Heidelberg. Springer-Verlag.

[5] Cano, J., Herrera, F., and Lozano, M. (2003). Using evolutionary algorithms as instance selection for data reduction in kdd: An experimental study. IEEE Transactions on Evolutionary Computation, 7(6):561-575.

[6] Cateni, S., Colla, V., and Vannucci, M. (2014). A method for resampling imbalanced datasets in binary classification tasks for real-world problems. Neurocomputing, 135:32-41.

[7] Chang, C. and Lin, C. (2011). Libsvm: a library for support vector machines. ACM Transactions on Intelligent Systems and Technology, 2(27):1-27.

[8] Chawla, N., Bowyer, K., Hall, L., and Kegelmeyer, W. (2002). Smote: Synthetic minority over-sampling technique. Journal Of Artificial Intelligence Research, 16:321-357.

[9] Chieslak, D., Chawla, N., and Striegel, A. (2006). Combating imbalance in network intrusion datasets. In Proocedings of the IEEE International Conference on Granular Computing, pages 732-737. 
[10] Cohen, G., Hilario, M., Sax, H., Hugonnet, S., and Geissbuhler, A. (2006). Learning from imbalanced data in surveillance of nosocomial infection. Artificial Intelligence in Medicine, 37:7-18.

[11] Eshelman, L. (1991). The chc adaptive search algorithm: How to have safe search when engaging in nontraditional genetic recombination. In Foundations of Genetic Algorithms, pages 265-283. Morgan Kaufmann.

[12] Fernández, A., García, S., Jesus, M., and Herrera, F. (2008). A study of the behaviour of linguistic fuzzy rule based classification systems in the framework of imbalanced data-sets. Fuzzy Sets and Systems, 159(18):2378-2398.

[13] García, S., Derrac, J., Triguero, I., Carmona, C., and Herrera, F. (2012a). Evolutionary-based selection of generalized instances for imbalanced classification. Knowledge-Based Systems, 25(1):3-12.

[14] García, S. and Herrera, F. (2009). Evolutionary undersmpling for classification with imbalanced datasets: proposals and taxonomy. Evolutionary Computation, 17(3):275-306.

[15] García, V., Sánchez, J., and Mollineda, R. (2012b). On the effectiveness of preprocessing methods when dealing with different levels of class imbalance. Knowledge-Based Systems, 25:13-21.

[16] García-Pedrajas, N., Pérez-Rodríguez, J., García-Pedrajas, M., Ortiz-Boyer, 
D., and Fyfe, C. (2012). Class imbalance mehtods for translation initiation site recognition in dna sequences. Knowledge-Based Systems, 25:22-34.

[17] Gu, Q., Cai, Z., Zhu, L., and Huang, B. (2008). Data mining on imbalanced data sets. In Proceedings of the International Conference on Advanced Computer Theory and Engineering, pages 1020-1024.

[18] Guo, H. and Viktor, H. (2004). Learning from imbalanced data sets with boosting and data generation: the databoost-im approach. SIGKDD Explorations, 6(1):30-39.

[19] Han, H., Wang, W., and Mao, B. (2005). Borderline-smote: A new oversampling method in imbalanced data sets learning. In Proceedings of the International Conference on Intelligent Computing (ICIC05), pages 878-887. Springer.

[20] Hart, P. (1968). The condensed nearest neighbor rule. IEEE Transactions on Information Theroy, 14:515-516.

[21] He, H., Bai, Y., García, E., and Li, S. (2008). Adasyn: Adaptive synthetic sampling approach for imbalanced learning. In Proceedings of International Joint Conference on Neural Networks (IJCNNO8), pages 1322-1328.

[22] He, H. and García, E. (2009). Learning from imbalanced data. IEEE Transactions on Knowledge and Data Engineering, 21(9):1263-1284. 
[23] Kubat, M. and Matwin, S. (1997). Addressing the curse of imbalanced training sets: one-sided selection. In Proceedings of the 14th International Conference on Machine Learning, pages 179-186.

[24] Laurikkala, J. (2001). Improving identification of difficult small classes by balancing class distribution. In Proceedings of the 8th Conference on AI in Medicine in Europe: Artificial Intelligence Medicine, AIME '01, pages 63-66, London, UK, UK. Springer-Verlag.

[25] Lin, Y., Lee, Y., and Wahba, G. (2002). Support vector machines for classification in nonstandard situations. Machine Learning, 46(1-3):191-202.

[26] Liu, Y., Chawla, N., Harper, M., Shriberg, E., and Stolcke, A. (2006). A study in machine learning from imbalanced data for sentence boundary detection in speech. Computer Speech and Language, 20:468-494.

[27] López, V., Fernández, A., García, S., Palade, V., and Herrera, F. (2013a). An insight into classification with imbalanced data: Empirical results and current trends on using data intrinsic characteristics. Information Sciences, 250:113141.

[28] López, V., Fernández, A., Jesus, M., and Herrera, F. (2013b). A hierarchical genetic fuzzy system based on genetic programming for addressing clas- 
sification with highly imbalanced and borderline data-sets. Knowledge-Based Systems, 38:85-104.

[29] Napierala, K., Stefanowski, J., and Wilk, S. (2010). Learning from imbalanced data in presence of noisy and borderline examples. In Proceedings of the 7th International Conference on Rough Sets and Current Trends in Computing (RSCT2010), pages 158-167.

[30] Peng, L., Qiao, P.-L., and Liu, Y.-C. (2008). A hybrid re-sampling method for svm learning from imbalanced data sets. In Fuzzy Systems and Knowledge Discovery, volume 2.

[31] Provost, F. (2000). Machine learning from imbalanced data sets 101. In Proceedings of the AAAI'2000 Workshop on Imbalanced Data Sets, pages 1-3.

[32] Provost, F. and Fawcett, T. (2001). Robust classification for imprecise environments. Machine Learning, 42(3):203-231.

[33] Quinlan, J. (1993). C4.5: Programs for Machine Learning. Morgan Kaufmann.

[34] Ramentol, E., Caballero, Y., Bello, R., and Herrera, F. (2012). Smote-rsb*: A hybrid preprocessing approach based on oversampling and undersampling for high imbalanced data-sets using smote and rough sets theory. Knowledge and Information Systems, 33:245-265. 
[35] Raskutti, B. and Kowalczyk, A. (2004). Extreme rebalanceing for svms: a case study. SIGKDD Explorations, 6(1):60-69.

[36] Sheskin, D. (2003). Handbook of parametric and nonparametric statistical procedures. Chapman \& Hall/CRC.

[37] Tomek, I. (1976). Two modifications of cnn. IEEE Transactions on Systems, Man and Cybernetics, 6:769-772.

[38] Weiss, G. and Provost, F. (2003). Learning when training data are costly: the effect of class distribution on tree induction. Journal Of Artificial Intelligence Research, 19:315-354.

[39] Wilson, D. (1972). Asymptotic properties of nearest neighbor rules using edited data. IEEE Transactions on Systems, Man and Cybernetics, 2(3):408421.

[40] Wong, G., Leung, F., and Ling, S. (2013). A novel evolutionary preprocessing method based on over-sampling and under-sampling for imbalanced datasets. In Proceedings of the 39th Annual Conference of the IEEE Industrial Electronics Society (IECON 2013), pages 2354-2359.

[41] Xu, L., Chow, M., and Taylor, L. (2007). Power distribution fault cause identification with imbalanced data using the data mining-based fuzzy classification e-algorithm. IEEE Transactions on Power Systems, 22(1):164-171. 
[42] Zhou, L. (2013). Performance of corporate bankruptcy prediction meodels on imbalanced dataset: The effect of sampling methods. Knowledge-Based Systems, 41:16-25. 